\title{
Synaptic Basis of Cortical Persistent Activity: the Importance of NMDA Receptors to Working Memory
}

\author{
Xiao-Jing Wang \\ Volen Center for Complex Systems and Department of Physics, Brandeis University, Waltham, \\ Massachusetts 02454-9110
}

Delay-period activity of prefrontal cortical cells, the neural hallmark of working memory, is generally assumed to be sustained by reverberating synaptic excitation in the prefrontal cortical circuit. Previous model studies of working memory emphasized the high efficacy of recurrent synapses, but did not investigate the role of temporal synaptic dynamics. In this theoretical work, I show that biophysical properties of cortical synaptic transmission are important to the generation and stabilization of a network persistent state. This is especially the case when negative feedback mechanisms (such as spike-frequency adaptation, feedback shunting inhibition, and short-term depression of recurrent excitatory synapses) are included so that the neural firing rates are controlled within a physiological range (10-50 Hz), in spite of the exuberant recurrent excitation. Moreover, it is found that, to achieve a stable persistent state, recurrent excitatory synapses must be dominated by a slow

Working memory is a fundamental cognitive function, by virtue of which information can be actively retained for seconds and used in the brain (Baddeley, 1986; Fuster, 1988; Goldman-Rakic, 1995). Its neuronal correlate, delay-period activity, has been widely documented by unit recording studies of behaving monkeys (Fuster and Alexander, 1971; Kubota and Niki, 1971; Miyashita and Chang, 1988; Gnadt and Andersen, 1988; Funahashi et al., 1989; Miller et al., 1996; Chafee and Goldman-Rakic, 1998; Rainer et al., 1998; Romo et al., 1999). For example, in a visuospatial delayed-response experiment (Funahashi et al., 1989), the animal's delayed saccadic eye movement is guided by the shortterm memory of a visual cue. Neurons in the dorsolateral prefrontal (PFC) cortex were found to display elevated firing activity during the entire delay period. This persistent activity is tuned to the spatial location of the cue in some cells, but not in other cells. Therefore, there are two distinct aspects of the mnemonic coding by the PFC cells: the persistent nature of the delay-period activity and the formation of the tuned "memory field".

It is generally assumed that persistent activity is sustained by some kind of reverberating discharge within a recurrent neural network (Hebb, 1949; Amit, 1995). The characteristic horizontal connections found in the superficial layers II-III of the dorsolat-

\footnotetext{
Received April 14, 1999; revised Aug. 12, 1999; accepted Aug. 12, 1999.

This work was supported by the National Science Foundation (IBN-9733006), the Alfred P. Sloan Foundation, and the W. M. Keck Foundation. I thank P. S. Goldman-Rakic, E. Marder, J. Lisman, and N. Brunel for discussions and helpful comments on this manuscript.

Correspondence should be addressed to Xiao-Jing Wang, Center for Complex Systems, Brandeis University, Waltham, MA 02454. E-mail: xjwang@volen. brandeis.edu.

Copyright (C) 1999 Society for Neuroscience 0270-6474/99/199587-17\$05.00/0
}

component. If neuronal firings are asynchronous, the synaptic decay time constant needs to be comparable to that of the negative feedback; whereas in the case of partially synchronous dynamics, it needs to be comparable to a typical interspike interval (or oscillation period). Slow synaptic current kinetics also leads to the saturation of synaptic drive at high firing frequencies that contributes to rate control in a persistent state. For these reasons the slow NMDA receptor-mediated synaptic transmission is likely required for sustaining persistent network activity at low firing rates. This result suggests a critical role of the NMDA receptor channels in normal working memory function of the prefrontal cortex.

Key words: working memory; prefrontal cortex; persistent activity; NMDA receptor; synaptic dynamics; short-term plasticity; rate control; synchronization; spiking neuron model

eral PFC may provide the anatomical substrate for such a recurrent circuit (Levitt et al., 1993; Kritzer and Goldman-Rakic, 1995). However, it remains unknown what are the realistic synaptic properties and circuit dynamics that are required for a robust network-induced persistent activity. Indeed, most previous model studies used simple firing-rate models (Wilson and Cowan, 1973; Amari, 1977; Zipser et al., 1993; Amit et al., 1994; Camperi and Wang, 1998; Moody et al., 1998). Amit and collaborators (Amit et al., 1990; Amit and Tsodyks 1991; Amit and Brunel, 1997) used leaky integrate-and-fire (LIF) spiking neurons but did not take into account realistic postsynaptic current time courses.

In this paper I present a network model of spiking neurons in which synapses are endowed with realistic gating kinetics, based on experimentally measured dynamical properties of cortical synapses. I will focus on how delay-period activity could be generated by neuronally plausible mechanisms; the issue of memory field formation will be addressed in a separate study. A main problem to be investigated is that of "rate control" for a persistent state: if a robust persistent activity necessitates strong recurrent excitatory connections, how can the network be prevented from runaway excitation in spite of the powerful positive feedback, so that neuronal firing rates are low and comparable to those of PFC cells $(10-50 \mathrm{~Hz})$ ? Moreover, a persistent state may be destabilized because of network dynamics. For example, fast recurrent excitation followed by a slower negative feedback may lead to network instability and a collapse of the persistent state. It is shown that persistent states at low firing rates are usually stable only in the presence of sufficiently slow excitatory synapses of the NMDA type. Functional implications of these results for the role of 
NMDA receptors in the PFC working memory function are discussed.

\section{MATERIALS AND METHODS}

The leaky integrate-and-fire model. To simulate a local recurrent cortical network, I used a network model of leaky integrate-and-fire neurons (Tuckwell, 1988), with either all-to-all or sparse connectivity. Such a network can be viewed as a cortical cell assembly that stores a particular memory item. As a result of Hebbian learning, the internal excitatory recurrent connections are strong and homogeneous, whereas the interactions between this cell assembly and the rest of the circuit are relatively weak and are neglected.

The network model consists of two populations of neurons $\left(N_{e}\right.$ pyramidal cells and $N_{i}$ inhibitory interneurons). Each pyramidal cell obeys the following equation:

$$
\begin{gathered}
C_{m} \frac{d V_{m}}{d t}=-I_{L}-I_{A H P}-I_{s y n, e e}-I_{s y n, i e}+I_{a p p} \\
\frac{d\left[\mathrm{Ca}^{2+}\right]}{d t}=\alpha_{C a} \sum_{j} \delta\left(t-t_{j}\right)-\left[C a^{2+}\right] / \tau_{C a}
\end{gathered}
$$

where $C_{m}$ is the capacitance, $I_{a p p}$ represents the afferent input, and the leak current $I_{L}=g_{L}\left(V_{m}-V_{L}\right)$. $I_{A H P}=g_{A H P}\left[\mathrm{Ca}^{2+}\right]\left(V_{m}-V_{K}\right)$ describes a calcium-activated potassium current for spike-frequency adaptation. $\left[\mathrm{Ca}^{2+}\right]$ is incremented by an amount $\alpha_{C a}$ with each spike discharge, and decays with a time constant $\tau_{C a}$ afterwards (cf. Treves, 1993; Y. H. Liu and X.-J. Wang, unpublished observations). $I_{\text {syn,ee }}$ and $I_{\text {syn,ie }}$ are the recurrent synaptic inputs from pyramidal cells and interneurons, respectively.

A spike is discharged each time $V_{m}$ is driven to reach a firing voltage threshold $V_{t h}$. Then $V_{m}$ is reset to $V_{\text {reset }}$ and stays there for an absolute refractory period $\tau_{\text {ref }}$. The intrinsic parameters were calibrated based on the intracellular data of cortical pyramidal neurons (McCormick et al., 1985; Mason and Larkman, 1990; Troyer and Miller, 1997): $C_{m}=0.5 \mathrm{nF}$, $g_{L}=0.025 \mu \mathrm{S}$ (so that the time constant $\tau_{m}=C_{m} / g_{L}=20 \mathrm{msec}$ ); $V_{L}=$ $-70, V_{\text {th }}=-52, V_{\text {reset }}=-59$ (in $\left.\mathrm{mV}\right) ; \tau_{\text {ref }}=2 \mathrm{msec}$. The frequencycurrent curve of an isolated cell has a current threshold $I_{c}=g_{L}\left(V_{t h}-\right.$ $\left.V_{L}\right)=0.45 \mathrm{nA}$. For the adaptation current $V_{K}=-85 \mathrm{mV}, \alpha_{C a}=0.2 \mu \mathrm{M}$, $\tau_{C a}=80 \mathrm{msec}$ (Helmchen et al., 1996), and $g_{A H P}$ will be specified in the text whenever it is not zero.

The interneuron model represents fast-spiking GABAergic cells that do not display spike-frequency adaptation (McCormick et al. 1985). Each interneuron obeys the equation:

$$
C_{m} \frac{d V_{m}}{d t}=-I_{L}-I_{s y n, e i}+I_{a p p}
$$

which is similar to Equation 1, except that $I_{A H P}$ is absent, and $I_{s y n, e i}$ is the recurrent synaptic input from pyramidal cells. Mutually inhibitory interactions among interneurons were not included. The parameter values for the interneurons are (cf. McCormick et al., 1985) $C_{m}=0.2 \mathrm{nF}, g_{L}=0.02$ $\mu \mathrm{S}\left(\tau_{m}=C_{m} / g_{L}=10 \mathrm{msec}\right) ; V_{L}=-65, V_{\text {th }}=-52, V_{\text {reset }}=-60(\mathrm{in} \mathrm{mV})$; $\tau_{\text {ref }}=1 \mathrm{msec}$. The frequency-current curve of an isolated interneuron has a current threshold $I_{c}=0.26 \mathrm{nA}$.

Synaptic kinetics and short-term depression. The EPSC originating from a presynaptic pyramidal cell consists of two components, $I_{A M P A}$ and $I_{N M D A}$. The AMPA receptor (AMPAR)-mediated current $I_{A M P A}=$ $g_{A M P A} s\left(V_{m}-V_{E}\right)$, with $V_{E}=0 \mathrm{mV}$. The gating variable $s$ (the fraction of open channels) is described by two first-order kinetics:

$$
\begin{gathered}
\frac{d x}{d t}=\phi\left(\alpha_{x} \sum_{j} \delta\left(t-t_{j}\right)-x / \tau_{x}\right) ; \\
\frac{d s}{d t}=\phi\left(\alpha_{s} x(1-s)-s / \tau_{s}\right),
\end{gathered}
$$

where the sum is over presynaptic spike times. The scaling factor $\phi$ controls the speed of synaptic kinetics without affecting the steady state, $\phi=1$ unless specified otherwise. For the AMPAR channels, I used $\tau_{x}=$ $0.05 \mathrm{msec}$ and $\tau_{s}=2 \mathrm{msec}$ (the time-to-peak is $\sim 0.2 \mathrm{msec}$ ); $\alpha_{x}=1$ (dimensionless), and $\alpha_{s}=1$ (in $\mathrm{msec}^{-1}$ ). The NMDA receptor (NMDAR)-mediated current $I_{N M D A}=g_{N M D A} s\left(V_{m}-V_{E}\right) /(1+$ $\left.\left[\mathrm{Mg}^{2+}\right] \exp \left(-0.062 V_{m}\right) / 3.57\right)$ (Jahr and Stevens, 1990), with a voltage dependence controlled by the extracellular magnesium concentration $\left[\mathrm{Mg}^{2+}\right]=1.0 \mathrm{~mm}$. The gating variable $s$ obeys the same types of equations (Eqs. 4, 5), but with $\tau_{x}=2 \mathrm{msec}$ and $\tau_{s}=80 \mathrm{msec}$ (the time-to-peak is $\simeq 8 \mathrm{msec}$ ).

This model of excitatory synapses was chosen for three reasons. First, it is based on a plausible kinetic scheme (Wang and Rinzel, 1992; Destexhe et al., 1994). In response to a presynaptic spike, the time course of $s$ has a smooth rising phase and an exponential decay with time constant $\tau_{E}=\tau_{s} / \phi$, that can be matched to the experimental data (Hestrin et al., 1990a; Lester et al., 1990). Second, there is temporal summation and, if the presynaptic firing frequency is high compared to $1 / \tau_{E}, s$ will saturate in the steady state $(s \leq 1)$ (Fig. 1). The saturation effect is much more significant for the slow NMDAR-mediated EPSC than for the fast AMPAR-mediated EPSC and has important implications for the network dynamical behavior. Finally, the model is sufficiently simple to allow detailed analysis of the network activity.

The IPSC originating from an interneuron is assumed to be mediated by $\mathrm{GABA}_{\mathrm{A}}$ receptors $\left(\mathrm{GABA}_{\mathrm{A}} \mathrm{Rs}\right), I_{G A B A}=g_{G A B A} s\left(V_{m}-V_{I}\right)$, with $V_{I}=$ $V_{L}=-70 \mathrm{mV}$ ("shunting inhibition"). The gating variable $s$ obeys a simple first-order kinetics with saturation (Wang and Rinzel, 1992):

$$
\frac{d s}{d t}=\alpha_{I} \sum_{j} \delta\left(t-t_{j}^{-}\right)(1-s)-s / \tau_{I},
$$

with $\alpha_{I}=0.9$ and $\tau_{I}=10 \mathrm{msec}$. The superscript in $t_{j}^{-}$indicates that the increment of $s$ by a spike should be calculated using the value of $s$ immediately before the spike on the right hand side of the equation, $\Delta s=$ $s\left(t_{j}^{+}\right)-s\left(t_{j}^{-}\right)=\alpha_{I}\left(1-s\left(t_{j}^{-}\right)\right)$.

Most simulations were done with all-to-all connectivity. In that case a neuron receives synaptic inputs from all neurons in the network, and the summation of synaptic currents is normalized by the number of neurons $N$. Sparse connectivity was also considered (see Fig. 11). There, the coupling between neurons is randomly assigned, with an average number of synapses per neuron $M_{s y n}$ (which is much smaller than $N$ ), and the summation of synaptic currents is normalized by $M_{s y n}$. The probability that a pair of neurons are connected in either direction is $p=M_{s y n} / N$.

In some of the model simulations, short-term depression was incorporated for the pyramid-to-pyramid recurrent excitatory synapses (Markram and Tsodyks, 1996; Abbott et al., 1997; C. M. Hempel, K. H. Hartman, X.-J. Wang, G. G. Turrigiano, and S. B. Nelson, unpublished observations). Short-term depression is assumed to be caused by transmitter vesicle depletion at the presynaptic terminals (Stevens and Wang, 1995). It is introduced into the synapse model as follows. The parameter $\alpha_{x}$, which mimicks the amount of transmitter release per spike, is multiplied by a quantity $D$ (the fraction of available vesicles). $D$ obeys the dynamical equation (Abbott et al., 1997):

$$
\frac{d D}{d t}=-p_{v} D \sum_{j} \delta\left(t-t_{j}^{-}\right)+(1-D) / \tau_{D}
$$

That is, $D$ is reduced by a factor $\left(1-p_{v}\right)$ for each spike, $\Delta D=D\left(t_{j}^{+}\right)-$ $D\left(t_{j}^{-}\right)=-p_{v} D\left(t_{j}^{-}\right)$, or $D\left(t_{j}^{+}\right)=\left(1-p_{v}\right) D\left(t_{j}^{-}\right)$. It recovers toward 1 with time constant $\tau_{D}$ in the absence of stimuli. In a simple biophysical model of vesicle depletion in which the release probability is proportional to the number of available vesicles, $p_{v}$ is identified with the release probability per vesicle (Wang, 1999). I used $\tau_{D}=500 \mathrm{msec}$ and $p_{v}=0-0.35$.

Asynchronous States. In this work, persistent activity is assumed to be achieved by a bistability between a rest state and an active state of the network. We shall see that the persistent activity often occurs as an asynchronous network state, in which the discharges of neurons are spread out in time uniformly so that at any time there is a same fraction of neurons firing (Amit and Tsodyks, 1991; Abbott and van Vreeswijk, 1993; Gerstner, 1999). In the presence of the voltage dependence of the NMDAR channels, the nonlinear LIF model cannot be solved explicitly, and the analysis of the asynchronous states is intractable. However, as we shall see, none of our conclusions in this work depends on the voltage sensitivity of the NMDAR-activated conductance. Therefore, the calculations of the asynchronous state were done with $\left[\mathrm{Mg}^{2+}\right]=0$.

The firing rates $R_{E}$ and $R_{I}$ of pyramidal cells and interneurons in an asynchronous state were calculated as follows. Let us denote the average synaptic drives by $s_{E}$ and $s_{I}$. Each of the two is an average over neural population, and is constant in time for an asynchronous state. It is the 

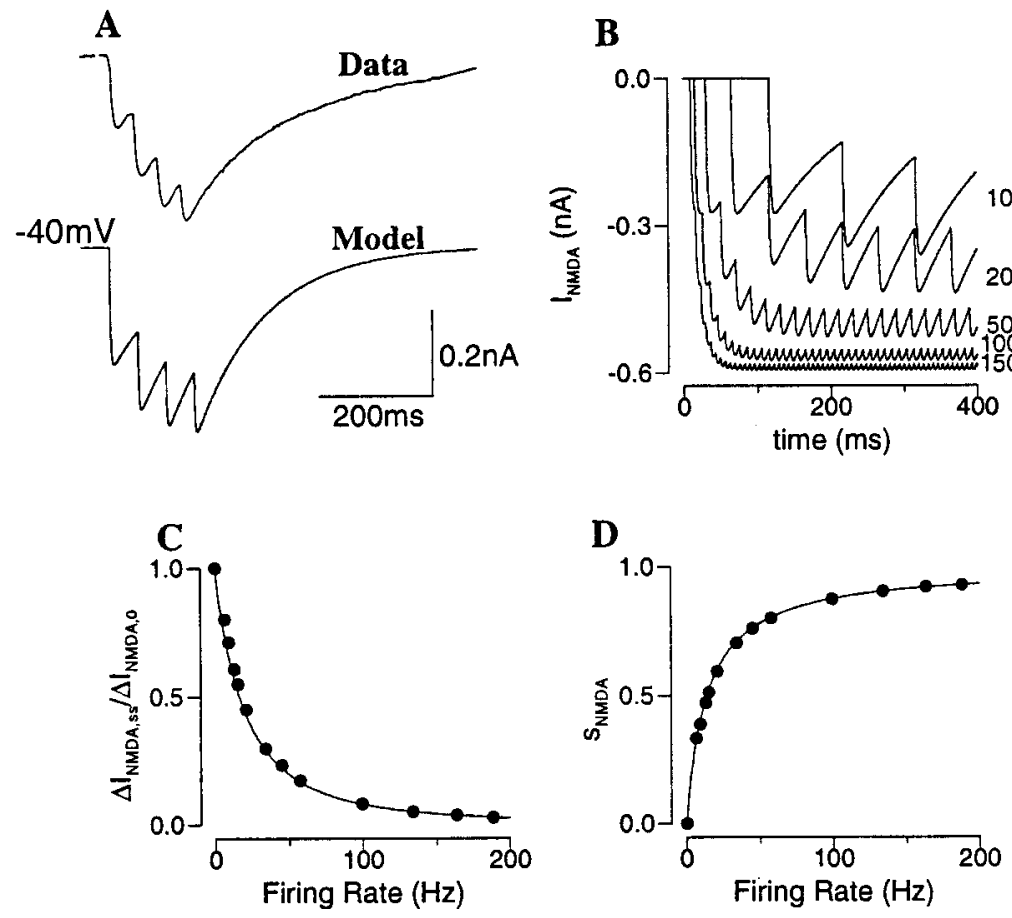

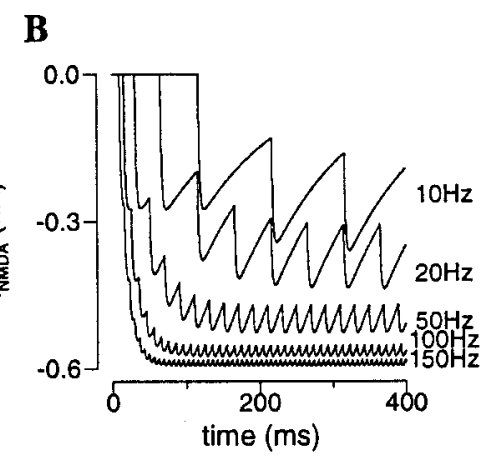

Figure 1. Temporal summation of the NMDAR-mediated EPSCs. $A$, NMDAR-mediated EPSCs elicited by four stimuli, when the membrane potential is clamped at $-40 \mathrm{mV}$. Top panel, Data from a pyramidal neuron in CA1 of the rat hippocampus (redrawn from Hestrin et al., 1990b, with permission). The stimulus is at $25 \mathrm{~Hz}$. Note the significant summation and saturation. These properties are mediated postsynaptically by the NMDARs, because they are absent in the non-NMDR-mediated EPSCs recorded in the same cell at $-100 \mathrm{mV}$. Bottom panel, NMDAR-mediated EPSCs produced by the model synapse (Eqs. 4, 5); the stimulus is at 20 Hz. $g_{N M D A}=0.07 ; \alpha_{x}=1, \tau_{x}=2 \mathrm{msec} ; \alpha_{s}=0.3, \tau_{s}=120$ msec. $B$, NMDAR-mediated EPSCs of the model synapse at various stimulus frequencies $R$. The EPSC amplitude decreases in time in each train, and its steady state is smaller at higher $R$. The average current saturates at high $R$. $C$, The ratio of the NMDAR-mediated EPSC in the steady state $\left(\Delta I_{N M D A}\right)$ over its initial value $\left(\Delta I_{N M D A, 0}\right)$, as function of the stimulus frequency. Solid curve, $A(R)=1 /(1+0.025 * R)^{2}$, which fits well the simulation data; therefore $\Delta I_{N M D A, s s} \sim$ $1 / R^{2}$ at high $R$. $D$, The average $s_{N M D A}$ as function of stimulus frequency. Solid curve, $s_{N M D A}=\nu R /(\nu R+1), \nu=\alpha_{x} \alpha_{s} \tau_{x} \tau_{s}$. same as the time average of each individual $s(t)$ over a period $1 / R$. For $s_{E}$ (Eqs. 4, 5), an approximation is obtained by substituting $\Sigma_{j} \delta\left(t-t_{j}\right)$ with $R$. The steady state is:

$$
x_{E}=\alpha_{x} \tau_{x} R_{E} ; \quad s_{E}=\frac{\alpha_{s} \tau_{s} x_{E}}{\alpha_{s} \tau_{s} x_{E}+1}=\frac{\nu R_{E}}{\nu R_{E}+1} .
$$

where $\nu=\alpha_{x} \alpha_{s} \tau_{x} \tau_{s} \mathrm{msec}^{-1}$. This approximation is accurate when the synaptic current kinetics are sufficiently slow (Ermentrout, 1994), hence reasonable for the NMDAR channels (Fig. 1D). On the other hand, it is also correct as long as saturation is negligible, which is the case for the fast AMPAR channels. The average $s_{E}$ only depends on the product $\nu$ and is independent of the scaling factor $\phi$. It becomes nonlinear in $R_{E}$ at $R_{E} \geq 1 / \nu$ and saturates at $R_{E} \gg 1 / \nu$. For AMPAR channels $\nu=0.1$ $\mathrm{msec}^{-1}, 1 / \nu=10 \mathrm{kHz}$; so $s_{A M P A}$ does not saturate at realistic firing rates, $s_{A M P A} \simeq \nu R_{E}$. For NMDAR channels $\nu=160 \mathrm{msec}^{-1}, 1 / \nu=6.25 \mathrm{~Hz}$, and $s_{N M D A}$ is a highly nonlinear function of $R_{E}$.

With short-term depression $\left(p_{v} \neq 0\right)$, the parameter $\alpha_{x}$ is multiplied by the steady-state value of $D$. It is worth noting that the amount of synaptic transmission is given by the value of $D$ immediately preceding a spike (denoted by $D_{-}$), and not the time average over a period. The steady state value of $D_{-}$is (Abbott et al., 1997; Wang, 1999):

$$
D_{-}=\frac{1-e^{-1 / R_{E} \tau_{D}}}{1-\left(1-p_{v}\right) e^{-1 / R_{E} \tau_{D}}} \simeq \frac{1}{p_{v} \tau_{D} R_{E}+1},
$$

where the approximation is obtained by $\Sigma_{j} \delta\left(t-t_{j}^{-}\right)=R_{E}$ in Equation 7 .

For the $\mathrm{GABA}_{\mathrm{A}} \mathrm{R}$-activated synaptic drive, the average was calculated over a periodic firing pattern of rate $R_{I}$ (compare Eq. 6):

$$
s_{I}=\frac{R_{I} \tau_{I} \alpha_{I}\left(1-e^{-1 / R I \tau I}\right)}{1-\left(1-\alpha_{I}\right) e^{-1 / R_{I} \tau_{I}}} .
$$

At realistic firing rates, the steady-state approximation obtained by $\Sigma_{j}(t-$ $\left.t_{j}^{-}\right)=R_{I}$ in Equation 6, $s_{I}=\alpha_{I} \tau_{I} R_{I} /\left(\alpha_{I} \tau_{I} R_{I}+1\right)$, is not accurate for the moderately slow IPSCs.

Given $s_{E}\left(R_{E}\right)$, the equation for an interneuron is the same as that of a single LIF neuron,

$$
C_{m} \frac{d V_{m}}{d t}=-g_{L}\left(V_{m}-V_{L}\right)-g_{s y n, i s} S_{E} V_{m}+I_{a p p}=-\tilde{g}_{L}\left(V_{m}-V_{\text {reset }}\right)+\tilde{I},
$$

with $\tilde{g}_{L}=g_{L}+g_{\text {syn,ei }} s_{E}$, and $\tilde{I}=I_{\text {app }}-g_{L}\left(V_{\text {reset }}-V_{L}\right)-g_{\text {syn }, e i} s_{E} V_{\text {reset }}$.
For a constant input current $I_{a p p}$, the firing rate is given by:

$$
R_{I}= \begin{cases}\frac{1}{\tau_{r e f}-\frac{C_{m}}{\tilde{g}_{L}} \ln \left[1-\left(\tilde{g}_{L} \theta\right) / \tilde{I}\right]} & \text { if } \tilde{I}>\tilde{g}_{L} \theta, \\ 0 & \text { otherwise }\end{cases}
$$

which is itself a function of $s_{E}\left(R_{E}\right)$ (i.e. the interneurons are driven by recurrent excitation). Similarly, the voltage equation for a pyramidal cell can be solved for a constant input current. The adaptation current has a steady-state average $I_{A H P}=g_{A H P}\left[\mathrm{Ca}^{+2}\right]_{a v}\left(V_{m}-V_{K}\right)$, where $\left[\mathrm{Ca}^{+2}\right]_{a v}=$ $\alpha_{C a} \tau_{C a} R_{E}$ according to Equation 2 with $\Sigma_{j} \delta\left(t-t_{j}\right)=R_{E}$. The same formula in Equation 12 applies to $R_{E}$, except that $\tilde{g}_{L}=g_{L}+$ $g_{A H P}\left[\mathrm{Ca}^{+2}\right]_{a v}+g_{\text {syn,ee }} s_{E}+g_{\text {syn,ie }} s_{I}$, and $\tilde{I}=I-g_{L}\left(V_{\text {reset }}-V_{L}\right)-$ $g_{A H P}\left[\mathrm{Ca}^{+2}\right]_{a v}\left(V_{\text {reset }}-V_{K}\right)-g_{\text {syn,ee }} s_{E} V_{\text {reset }}-g_{\text {syn,ie }} s_{I}\left(V_{\text {reset }}-V_{I}\right)$.

In simulations, noise was added by including a random component $I_{\lambda}=$ $i_{\lambda} s_{\lambda}$ in the external current, $I_{a p p}=I_{0}+I_{\lambda} ; I_{0}$ is a constant current, and $I_{\lambda}$ is a stochastic synaptic current of the AMPA type. With a Poisson input train of rate $\lambda, s_{\lambda}$ is incremented by 1 with each input and decays with a time constant $\tau_{\lambda}=2 \mathrm{msec}$. At a high rate $\lambda$, this Poisson current is approximated by a Gaussian white noise with a mean $\mu=i_{\lambda} \lambda \tau_{\lambda}$ and a variance $\sigma^{2}=i_{\lambda}^{2} \tau_{\lambda} \lambda$. Unless noted otherwise, $i_{\lambda}=0.06 \mathrm{nA}$ and $\lambda=2500$ $\mathrm{Hz}$ for pyramidal cells; and $i_{\lambda}=0.04 \mathrm{nA}$ and $\lambda=2000 \mathrm{~Hz}$ for interneurons. Given a fixed $\mu=0.06 \times 2.5 \times 2=0.3 \mathrm{nA}$, the mean input current to pyramidal cells $I=I_{0}+\mu$ can be varied by changing $I_{0}$, whereas the noise amplitude remains the same.

In the presence of noise, the neural discharges are described by the first-passage times across the firing threshold (Ricciardi, 1977), instead of Equation 12. The expression for the firing rate is:

$$
1 / R=\tau_{r e f}+\sqrt{\pi} \tilde{\tau}_{m} \int_{C_{m}\left(V_{\text {reset }}-V_{s s}\right) /\left(\sqrt{\tau_{m}} \sigma\right)}^{C_{m}\left(V_{t h}-V_{s s}\right) /\left(\sqrt{\tau_{m}} \sigma\right)} d x e^{x^{2}}(1+\operatorname{erf}(x)),
$$

where $\operatorname{er} f(x)=(2 / \sqrt{\pi}) \int_{0}^{x} \exp \left(-x^{\prime 2}\right) d x^{\prime}$ is the error function, $V_{s s}=I_{e f f} / \tilde{g}_{L}$ and $\tilde{\tau}_{m}=C_{m} / \tilde{g}_{L}\left(\tilde{g}_{L}\right.$ as given above $)$. The effective current $I_{e f f}=I_{0}+\mu+$ $g_{L} V_{L}$ for interneurons, and $I_{e f f}=I_{0}+\mu+g_{L} V_{L}+g_{A H P}\left[\mathrm{Ca}^{+2}\right]_{a v} V_{K}+$ $g_{\text {syn,ie }} s_{I} V_{I}$ for pyramidal cells.

The neural firing rates of the asynchronous state were approximately computed in two steps. First, Equation 13 applied to $R_{I}$ is a function of $R_{E}, R_{I}=g\left(R_{E}\right)$. Then, Equation 13 for $R_{E}$ becomes self-consistent,

$$
R_{E}=f\left(R_{E}, R_{I}\right)=f\left(R_{E}, g\left(R_{E}\right)\right) ; \quad R_{I}=g\left(R_{E}\right),
$$


which is solved to yield $R_{E}$. Note that $f$ is the input-output function of a LIF neuron, another way of writing Equation 14 is:

$$
R_{E}=f\left(I_{t o t}\right), \quad \text { where } I_{t o t}=I_{a p p}-I_{A H P}-I_{s y n, e e}-I_{s y n, i e},
$$

where $I_{t o t}$ depends on $I_{a p p}, R_{E}$ and $R_{I}$.

In numerical simulations, the initial condition can be prescribed to be near the asynchronous state, by assuming that the neural output patterns are periodic with the phases uniformly distributed in a time period $[0$, $T=1 / R]$ (Abbott and van Vreeswijk, 1993). Note that in the presence of noise, the time course of the neural membrane potential is not exactly periodic. However, this initial condition should be close to the actual asynchronous state. If the latter is a stable attractor, with this initial condition the network should quickly converge to it.

Numerical integrations. The model was numerically integrated using a second order Runge-Kutta method, with an interpolation procedure to determine the spike times (Hansel et al., 1998). The time step dt $=$ $0.02-0.05$ msec. Typically I used $N_{e}=1000$ and $N_{i}=200$, some conclusions were checked with $N_{e}=5000$ and $N_{i}=1000\left(N_{i} / N_{e}=20 \%\right)$.

In simulations, the network activity was measured by the instantaneous firing rate $R_{E}(t)$ of the pyramidal cell population as follows. The time was divided into small bins ( $\Delta t=1-10$ msec were used). Then,

$R_{E}(t)=\frac{\text { total number of spikes from all pyramidal cells in }(t, t+\Delta t)}{N_{e} \Delta t}$.

For example, in an asynchronous state $R_{E}$ would be constant in time and equals the firing rate of each individual cell. A coherent network oscillation would be reflected by a rhythmic time course of $R_{E}(t)$.

\section{RESULTS}

\section{NMDA receptor channels and persistent activity at low rates}

Persistent activity is produced by an excitatory neural network, when the recurrent synapses are sufficiently strong. In Figure $2 A$, the network is initially in a rest state. In response to a transient input pulse, neurons start to discharge spikes that activate recurrent synapses, which in turn elicit more spikes. This positive feedback loop between the spike firing and the recurrent synaptic drive leads to a self-sustained network activity, outlasting the input. In the persistent state, neurons fire spikes asynchronously in time: at any given moment there is always a fixed fraction of cells firing. Therefore, the synaptic drive to each cell is tonic (constant in time). Moreover, the average firing rate of neurons is $\sim 40 \mathrm{~Hz}$, within the physiological range of the persistent activity of PFC cells during the delay period (Funahashi et al., 1989; Rainer et al., 1998). The network is turned off by a brief hyperpolarizing input, from the persistent state back to the rest state. In this simulation, the leak conductance $g_{L}$ differs from cell to cell according to a Gaussian distribution. Cells with the smallest $g_{L}$ values are the most excitable and display spontaneous firing in the rest state; whereas cells with the largest $g_{L}$ values are the least excitable and only show transient responses to the input pulse but no persistent activity (Fig. $2 A$ ).

The bistability between the rest state and active state is a network phenomenon. As illustrated in Figure $2 B$, during persistent activity, a neuron can be temporally hyperpolarized by an applied current pulse, but its activity resumes itself immediately after the perturbation, because the firing of any single neuron is sustained by synaptic inputs from the circuit. Such a manipulation would be feasible experimentally only with intracellular recording from a behaving animal during a working memory task. The prediction is that if bistability is not a single cell property but is instead induced by the network circuit, a hyperpolarizing current pulse should be incapable of switching a neuron off from its persistent activity.

In model simulations, the NMDAR-mediated synaptic trans-
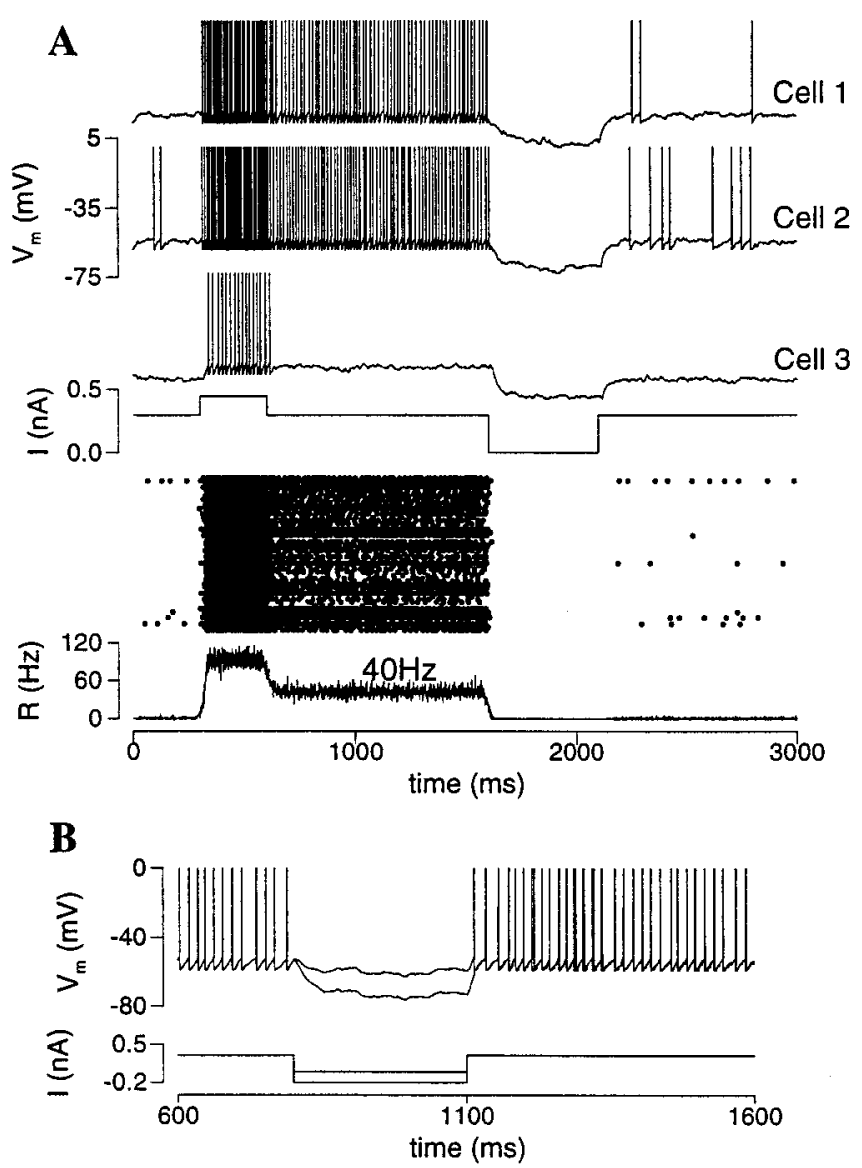

Figure 2. Persistent active state in an excitatory neural network. $A$, Panels from top to bottom, membrane potentials of three cells, external input current, rastergram, and population firing rate. The network model is initially at rest. In response to a transient current pulse, the network is activated. After the termination of the input, neurons continue to discharge spikes asynchronously with an average firing rate of $40 \mathrm{~Hz}[R(t)$ is constant in time; see also the rastergram]. In this simulation, there is a Gaussian distribution of the leak conductance $g_{L}$ across the cell population, with a mean of $0.025 \mu \mathrm{S}$ and SD of $0.003 \mu \mathrm{S}$. Cells with the least $g_{L}$ display spontaneous firing in the rest state (Cells 1,2), whereas cells with the largest $g_{L}$ do not show sustained firing in the network persistent state (Cell 3) $\left(g_{A M P A}=0.2 ; g_{N M D A}=0.04 ; I=0.3 \mathrm{nA}\right)$. B, Bistability is a network phenomenon. During persistent activity, a neuron is hyperpolarized by a current pulse (with two different intensities) to a negative membrane potential, but at the end of the perturbation the firing activity resumes itself because of the massive synaptic drive from the network.

mission was necessary to generate network persistent activity, at low firing rates such as in Figure 2. For the purpose of illustration, consider first the simplified situation of a perfectly synchronous network state in which all neurons behave exactly the same in time. Therefore, the population of identical excitatory neurons can be reduced to a single neuron endowed with an autapse (Fig. $3 A$ ). Suppose that the synaptic transmission is of the NMDA type (decay time $\tau_{E}=80 \mathrm{msec}$ ). The cell is switched onto a firing state by a transient input. At the end of input pulse, the NMDARmediated current decays slowly, and after the time span of an interspike interval (ISI), it remains large enough to trigger another spike, which in turn generates more EPSC. This process between the spiking and synaptic activation can continue indefinitely, provided that the decay of the NMDAR-mediated current is not too fast compared to a typical ISI, i.e. the $\tau_{E} / I S I$ ratio is sufficiently large. Otherwise, if the synaptic current generated by 

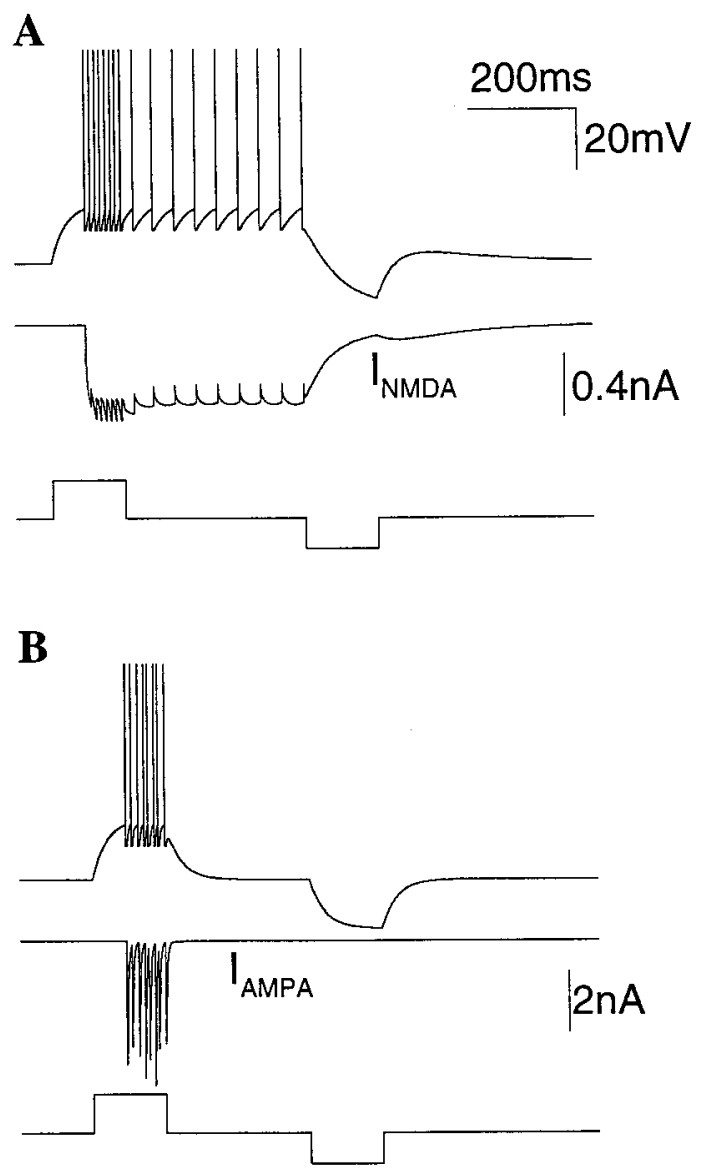

Figure 3. Tonic synaptic drive is required to sustain a persistent active state. $A$, A single neuron with an autapse of the NMDA type is excited from the rest to an active state that outlasts the transient input. The persistent firing is at $36 \mathrm{~Hz}$. Note the tonic NMDAR-mediated current $\left(g_{N M D A}=0.1\right)$. B , If the synaptic current is mediated by the AMPARs $\left(g_{A M P A}=1.5\right)$, the synaptic current fluctuates rapidly between a maximum and zero. When it is zero, the cell does not receive synaptic drive any more; therefore the cell decays back to the rest state as soon as the input is withdrawn. Note the different scale for the synaptic current in $A$ and $B$.

one spike decays back to zero before the next spike is triggered, the cell will return to the rest state instead. This is shown in Figure $3 B$, where the synapse is now assumed to be of the AMPA type $\left(\tau_{E}=2 \mathrm{msec}\right)$. The peak AMPAR-mediated EPSC here is $\sim 10 \times$ that of the NMDAR-mediated EPSC in Figure $3 A$, but it decays rapidly between spikes during the input pulse and does not give rise to persistent activity. Using a considerably stronger synaptic conductance, the AMPAR-mediated current can be large enough to generate a persistent activity, but at a very high firing rate, so that the $\tau_{E} / I S I$ ratio is again large (see below).

The above argument applies to the network, if the neural firing patterns are partially synchronous. For example, this can happen because of the interplay between rapid synaptic excitation and slower inhibition in a two-population network of pyramidal cells and interneurons (Fig. 4). Powerful AMPAR-activated synapses between pyramidal cells amplify the network activity, which is damped afterwards by recurrent inhibition, leading to synchronous network oscillations at $\sim 8 \mathrm{~Hz}$ (the oscillation frequency ranges from 8 to $65 \mathrm{~Hz}$, when the pyramid-to-interneuron coupling strength is varied gradually). Note that the AMPARactivated synaptic drive $s_{A M P A}$ fluctuates between zero and a peak level during the oscillation. Without NMDAR channels, clearly

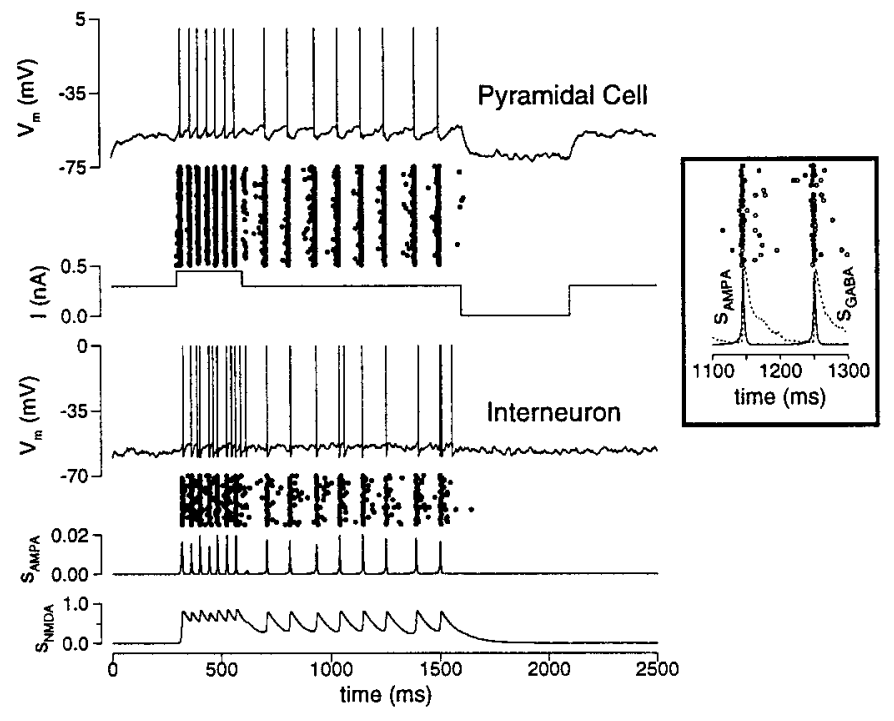

Figure 4. Slow NMDAR channels can sustain a persistent active state in which the network dynamics is partially synchronous. The network model consists of two (pyramid and interneuron) populations. The network is initially at rest and is switched to the active state by a transient input. Synchronous oscillations at $8 \mathrm{~Hz}$ are generated by the interplay between the fast recurrent AMPAR-activated excitation and slower feedback inhibition. Note that the pyramidal cell and interneuron populations show very small relative phase shift (inset). The AMPAR-activated synaptic drive $s_{A M P A}$ phasically oscillates between zero and a maximum, whereas the NMDAR-activated synaptic drive $s_{N M D A}$ remains at a significantly high level, which is sufficient to maintain the network activity $\left(g_{A M P A, e e}=0.7 ; g_{N M D A, e e}=0.07 ; g_{A M P A, e i}=0.2 ; g_{N M D A, e i}=0.02 ;\right.$ $\left.g_{\text {GABA }}=0.1 ; I=0.3 \mathrm{nA}\right)$.

this synchronous network state would not be self-sustained, because when $s_{A M P A}$ is almost zero the network would have to collapse back to the rest state. The slow NMDAR-mediated current does not decay back to zero during the waning phases of the network oscillation. As a result, the tonic component of the NMDAR-activated synaptic drive $s_{N M D A}$ can sustain a synchronous persistent state. Here, the requirement is that the oscillation period $T$ must not be too long compared to the NMDAR channel decay time constant $\left(\tau_{E} / T\right.$ must be large).

Therefore, by virtue of its temporal summation, NMDAR channels (but not AMPAR channels) can provide sufficient tonic drive to maintain a synchronous persistent state at low rates. On the other hand, if the persistent state is asynchronous, a tonic synaptic drive can be realized by a spatial summation over neurons. In the latter case, because the synaptic drive is constant in time regardless of the $\tau_{E} / I S I$ ratio, it would seem that the fast AMPAR channels alone might be sufficient to maintain a persistent network state at any firing rate. As we will see below, this is not the case because of the problem of rate control with the AMPAR channels.

\section{Frequency-current relation of a bistable network}

Persistent activity in our network model is realized as a bistability between a rest state and an active state, where the network can be switched on from the rest state by a transient stimulus and remains in the persistently active state afterwards. Consider for example the case where synaptic connections are mediated by the fast AMPARs. For a fixed synaptic coupling $\left(g_{A M P A}=1.05\right)$ and a given external drive $(I=0.3 \mathrm{nA})$, the neuronal firing rate of an asynchronous network is given by the nonlinear equation $R=$ $f\left(I_{t o t}(R)\right)$ (Eq. 15 in Materials and Methods). The function $f$ is the 
Figure 5. Frequency-current relation for a bistable network of pyramidal neurons. $A$, Bistability with AMPAR-activated synaptic drive $\left(g_{A M P A}=1.05\right)$. Top panel, For a fixed external input drive, the population firing rate of the asynchronous state is given by $R=f(R)$. Such states are obtained graphically by the intersections of the function $f(R)$ with the diagonal line. There are three states for $I=0.3$ (solid curve); two (rest and active) states are stable (filled circles), and one is unstable (open circle). If $I$ is too small $(I=0.1$; dotted line $)$ or too large $(I=0.5$; dashdotted line), there is only one steady state that is resting or active, respectively. Bottom panel, Bistability is manifested by the presence of three branches of the frequency-current curve; the bottom branch is the rest state, the top branch is the active state, and the middle branch is unstable. Within a range of external input current, denoted by $I_{a}$ and $I_{b}$, the network can be either at rest or in the active state. $B$, Different frequency-current curves correspond to $g_{A M P A}=0.6$ to 1.5 , by increment of 0.15 . With larger $g_{A M P A}$ the bistable range $\left(I_{b}-I_{a}\right)$ is wider, but the lowest firing rate of the active state located at $I_{a}$ (filled square) is dramatically increased. $C$, Bistability with NMDARactivated synaptic drive $\left(g_{N M D A}=0.006\right)$. Top panel, For a fixed $I=0.3 \mathrm{nA}$, with NMDAR channels the function $f(R)$ shows a plateau at relatively low $R$ values, because of the saturation of the NMDAR-activated conductance (compare Fig. 1), yielding a relatively low firing rate of the persistent state. Bottom panel, Frequency-current curve. D, Different frequency-current curves correspond to $g_{N M D A}=0.0$ to 0.014 by increment of 0.002 (the asynchronous state was calculated with $\left.\left[\mathrm{Mg}^{2+}\right]=0\right)$. With larger $g_{N M D A}$ the bistable range is wider $\left(I_{a}\right.$ is shifted to the left), whereas the minimal firing rate of the persistent state (filled square) remains $<40 \mathrm{~Hz}$.
AMPA
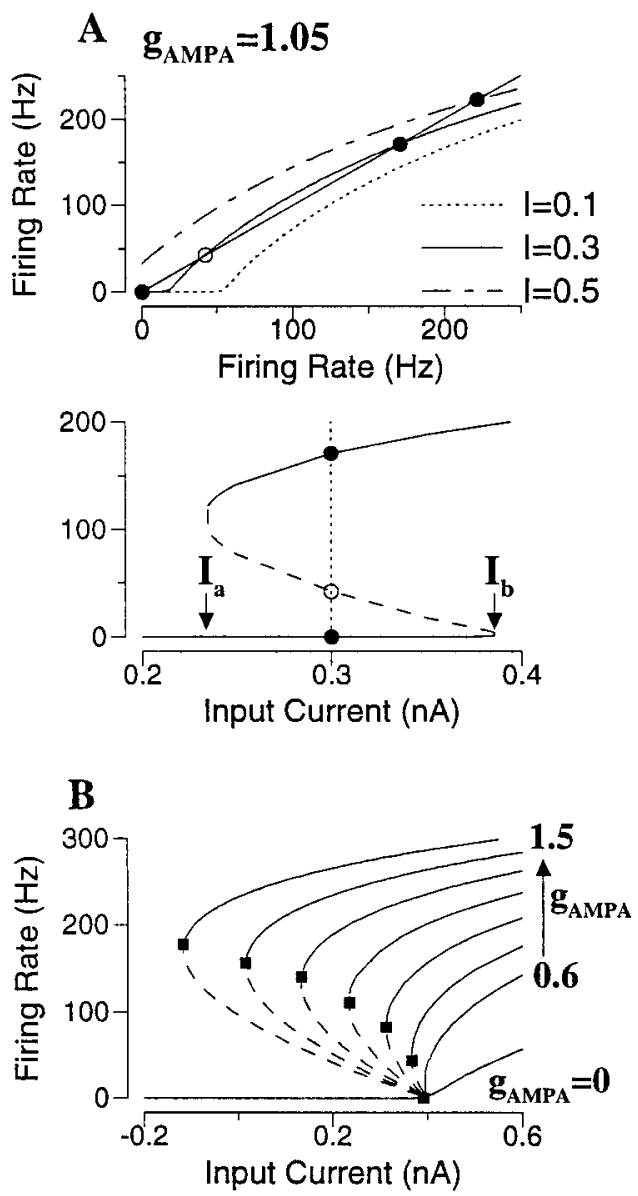

NMDA
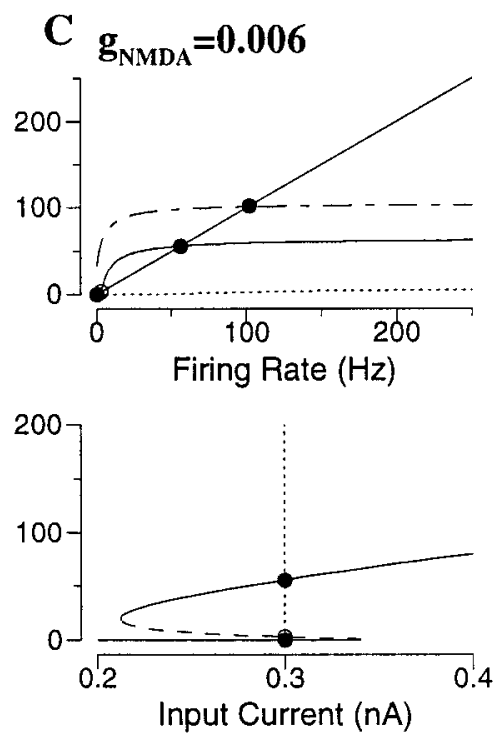

D

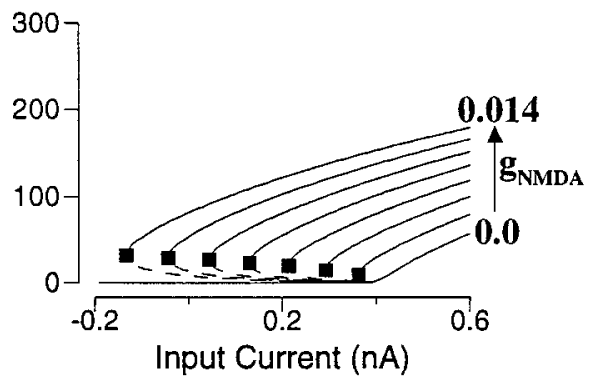

neuronal input-output relation, and the total input $I_{\text {tot }}$ is a function of $R$ caused by the recurrent synaptic interactions. When the left and right hand sides of the equation are plotted on a same graph, the solutions for $R$ correspond to the intersection points of the two curves. As shown in Figure $5 A$ (top panel, solid curve), there are three states of different firing rates: a rest state (in which synapses are not activated), an active (persistent) state, and a middle state (which is always unstable, thus not observable in network simulations). The instability of a steady state can be intuitively understood as follows. When $f\left(I_{t o t}\right)<R$, the total current acts to decrease firing, whereas when $f\left(I_{t o t}\right)>R$ the total current acts to increase firing. Therefore, if the rate $R$ happens to be slightly higher than the middle steady state, $f\left(I_{\text {tot }}\right)>R$ and $R$ will increase further; whereas if $R$ is lower than the middle state, $f\left(I_{\text {tot }}\right)<R$ and $R$ will decrease further. In either case the system will drift away, and the middle steady state is not stable against small perturbations.

The bistability occurs within a certain range of the $I$ values (Fig. $5 A$, top panel ). If the external drive is too small $(I=0.1)$, the combined external and recurrent drive is not sufficient to maintain a persistent state. On the other hand, if it is too large $(I=$ $0.5)$, the rest state no longer exists. By plotting the steady states as function of $I$, an S-shaped frequency-current curve is obtained for a bistable asynchronous network (Fig. 5A, bottom panel). Let us denote by $I_{a}$ and $I_{b}$ the two $I$ values delimiting the bistable range. $I_{a}$ is the smallest $I$ value for an active state, and $I_{b}$ is the largest $I$ value for the rest state. $I_{b} \simeq 0.4 \mathrm{nA}$ is close to the threshold current for an isolated neuron, because recurrent synapses are not activated in the rest state. The firing rate of the active state increases with $I$; the lowest possible rate corresponds to $I_{a}$, at the left-knee of the curve. In our example neuronal firing rates of persistent activity are above $110 \mathrm{~Hz}$, much higher than those observed in the PFC neurons $(10-50 \mathrm{~Hz})$.

Can the firing rate of persistent activity be reduced by weaker recurrent synaptic connections? In Figure $5 B$ are shown the frequency-current curves of the network at various coupling strengths $\left(g_{A M P A}\right)$. We see that bistability becomes possible only with sufficiently strong $g_{A M P A}$. With larger $g_{A M P A}$, persistent state can be realized at smaller $I\left(I_{a}\right.$ shifts to the left $)$, so the bistability range $\left(I_{b}-I_{a}\right)$ is wider (the persistent state is more robust). On the other hand, the lowest firing rate of a persistent state (at $I_{a}$ ) dramatically increases with $g_{A M P A}$ (Fig. $5 B$, filled square). Therefore, there is a tradeoff between the lowest firing rate possible and the robustness of the phenomenon: if we require that the bistable range be reasonably large (at least $0.1-0.3 \mathrm{nA}$, for example), the firing rate of a persistent state is always $100-200 \mathrm{~Hz}$ or higher. Furthermore, the stability of the active state is not guaranteed. Indeed, the persistent state close to $I_{a}$ is usually not observed in 
direct simulations of the network model, presumably because it is not stable in the presence of noise. The stability issue will be discussed in more detail below, when negative feedback processes are included.

In contrast to the case with AMPAR-activated synaptic transmission, with only NMDAR-activated synaptic transmission, robust persistent states at low firing rates are possible (Fig. 5C,D). The bistable range increases nearly linearly with the NMDARactivated conductance $g_{N M D A}$, whereas the lowest firing rate of the persistent state remains $<40 \mathrm{~Hz}$ (Fig. 5D, filled square). The dramatically different input-output relations obtained with the AMPA- or NMDA-type synapses can be explained in terms of their respective gating kinetics. As shown in Figure $5 C$ (top panel $)$, for a given synaptic coupling $\left(g_{N M D A}=0.006\right)$ and external drive $(I=0.3 \mathrm{nA})$, the input-output relation $f(R)$ saturates at low firing rates with NMDAR channels, in contrast to the case with AMPAR channels (Fig. 5A, top panel). This is because the dependence of $f$ on $R$ is via the synaptic drive $s_{E}(R)$ (Eq. 8). The fast-decaying AMPAR channels do not accumulate over time, hence do not saturate except at very high firing rates $(\sim 500 \mathrm{~Hz})$. By contrast, the slowly decaying NMDAR-mediated current saturates at firing rates within the physiological range (Fig. 1D). At $>50 \mathrm{~Hz}$ or so $s_{N M D A}$ becomes independent of the input rate, so it can no longer be increased further to sustain higher firing rates. (The actual firing rate, which also depends on $g_{N M D A}$ and the input $I$, can of course be $>50 \mathrm{~Hz}$.) For this reason, NMDAR (not AMPAR) channels are well suited to realize persistent states at low firing rates in a robust manner.

\section{Negative feedback mechanisms for rate control}

Can some negative feedback mechanisms be used to resolve the problem of rate control with the AMPAR channels alone? This question is addressed next, by considering consecutively spikefrequency adaptation, recurrent shunting inhibition, and shortterm synaptic depression.

\section{Spike-frequency adaptation}

Spike-frequency adaptation, a common property of ("regular spiking") cortical pyramidal neurons (McCormick et al., 1985; Mason and Larkman, 1990; Wang, 1998), is added to the model neuron by including an $I_{A H P}$. To assess the effects of $I_{A H P}$ on a persistent state sustained by the AMPAR channels, the frequencycurrent curve is calculated for different $g_{A H P}$ values (Fig. $6 A$ ). For a fixed $I$ the firing rate of the active state is reduced by $g_{A H P}$, (Fig. $6 A$, vertical dotted line). At the same time, however, the bistable range shrinks dramatically and eventually disappears with large $g_{A H P}$ values (for $g_{A H P} \geq 0.005$ ).

This effect of $I_{A H P}$ is readily explained in term of a negative current that counterbalances the excitatory synaptic current. Suppose that the firing rate is given by the input-output relation $R=$ $f\left(I_{t o t}\right)$ (Eq. 15), where $I_{t o t}=I_{a p p}-I_{\text {syn }}-I_{A H P}$. The average membrane potential of a firing neuron is approximately half-way between $V_{\text {reset }}$ and $V_{t h}, V_{a v} \simeq\left(V_{\text {reset }}+V_{\text {th }}\right) / 2=-55.5 \mathrm{mV}$. Then, one has $I_{s y n} \simeq g_{A M P A} s_{E} V_{a v} \simeq g_{A M P A v} V_{a v} R=-\tilde{g}_{A M P A} R$, with $s_{E} \simeq$ $v R$ and $\tilde{g}_{A M P A}=-g_{A M P A v} V_{a v}$. On the other hand, $I_{A H P} \approx g_{A H P}$ $\left[\mathrm{Ca}^{2+}\right]_{a v}\left(V_{a v}-V_{K}\right)=\tilde{g}_{A H P} R$, with $\left[\mathrm{Ca}^{2+}\right]_{a v}=\alpha_{C a} \tau_{C a} R$ and $\tilde{g}_{A H P}=g_{A H P} \alpha_{C a} \tau_{C a}\left(V_{a v}-V_{K}\right)$. Taken together, we have

$$
I_{t o t}=I_{a p p}+\left(\tilde{g}_{A M P A}-\tilde{g}_{A H P}\right) R .
$$

Therefore, the addition of $I_{A H P}$ amounts to a subtractive reduction of the effective recurrent synaptic excitation. For example, if $g_{A M P A}=1.2$ and $g_{A H P}=0.0025, \tilde{g}_{A M P A}=-g_{A M P A} \nu V_{a v}=$
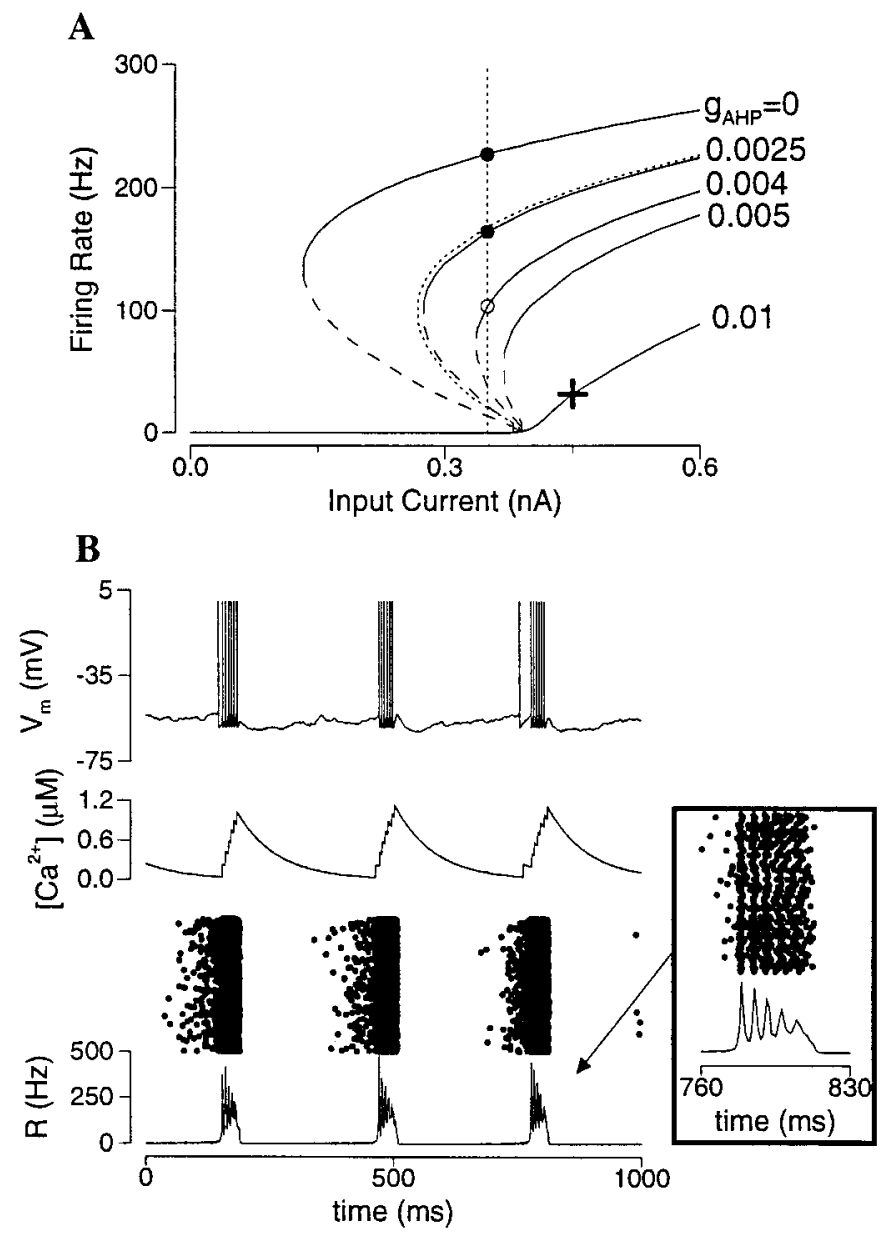

Figure 6. Effect of spike-frequency adaptation in an excitatory network $\left(g_{A M P A}=1.2\right) . A$, Frequency-current curves with different $g_{A H P}$ values. For a given input current (e.g., $I=0.35 \mathrm{nA}$; vertical dotted line), the firing rate is decreased by increasing $g_{A H P}$. At the same time, the bistable range shrinks, and the bistability disappears when $g_{A H P}$ is $>0.005$. Dotted line, $g_{A M P A}=0.99$ and $g_{A H P}=0$, which is superimposable with that of $g_{A M P A}=$ 1.2 and $g_{A H P}=0.0025$. The persistent state at reduced firing rate (e.g. open circle at $I=0.35$ and $g_{A H P}=0.004$ ) is unstable if the excitatory synapses are mediated by the fast AMPARs (see Appendix). B, Adaptation induced network rhythmic bursting. When the asynchronous state is unstable and does not coexist with the rest state, the network displays synchronous burst firing patterns (with $I=0.45$ and $g_{A H P}=0.01$, indicated by a cross in $A$ ). Strong and fast recurrent excitation recruits neurons and accelerates neural discharges, until $I_{A H P}$ grows sufficiently to terminate the burst. $I_{A H P}$ then decays back to zero, and the cycle starts over again. Note that the neural firing is coherent at the onset of the burst, but desynchronizes within the burst (inset).

6.66 and $\tilde{g}_{A H P}=g_{A H P} \alpha_{C a} \tau_{C a}\left(V_{a v}-V_{K}\right)=1.18$. Thus, $\tilde{g}_{A M P A}-$ $\tilde{g}_{A H P}=6.66-1.18=5.48$. This is equivalent to a reduced $g_{A M P A}$ value $\left(\tilde{g}_{A M P A}-\tilde{g}_{A H P}\right) /\left(-\nu V_{a v}\right)=0.99$ in the absence of $I_{A H P}$. Indeed, the frequency-current curve with $g_{A M P A}=1.2$ and $g_{A H P}=0.0025$ and that with $g_{A M P A}=0.99$ and $g_{A H P}=0$ are essentially superimposable (Fig. $6 A$ ).

Note that the specific form of this subtraction depends on the model details. For example, if $I_{A H P}$ has the following functional form $I_{A H P}=g_{A H P}\left[\mathrm{Ca}^{2+}\right]^{n} /\left(\left[\mathrm{Ca}^{2+}\right]^{n}+D_{K}^{n}\right)\left(V_{m}-V_{K}\right), n>1$, then the subtractive term in Equation 16 will be nonlinear.

It is important to emphasize that the stability of an active state is not guaranteed. In the Appendix it is shown that the stability of an asynchronous state depends critically on the synaptic time 
constant. In fact, with the fast AMPAR-mediated synapses, any active state in the presence of an $I_{A H P}$ is expected to be unstable if its firing rate is below the lowest possible firing rate of an active state with $I_{A H P}=0$. This is true regardless whether the active state belongs to a bistable range or not. For example, at $I=0.45$ $\mathrm{nA}$ and $g_{A H P}=0.01$ there is a single state with $R=30 \mathrm{~Hz}$ (Fig. $6 A$, cross). As shown in Figure $6 B$, this asynchronous state is not stable. Instead, neurons fire synchronously repetitive bursts of spikes that alternate with quiescent phases in time, the network oscillation has a frequency of $3 \mathrm{~Hz}$. Such rhythmic bursting has also been reported in other studies that are not related to persistent activity (van Vreeswijk and Hansel, 1997; G. B. Ermentrout, personal communication). Synchronous burst oscillation is a common phenomenon in neurons and networks, usually when a strong and rapid autocatalytic process is combined with a slower negative feedback (here, the recurrent AMPAR-activated synaptic excitation and the $\left.I_{A H P}\right)$. Clearly, because the fast AMPARactivated synaptic drive goes to zero between the bursts, the network would have to collapse onto the rest state, if the latter existed. In other words, when the active state in a bistable range is unstable (Fig. 6A, open circle), it is not observable, and the only stable behavior is the rest state. From these results it is concluded that $I_{A H P}$ cannot subserve as a rate control mechanism unless additional slow synaptic transmission is present, such as that mediated by the NMDARs.

\section{Recurrent shunting inhibition}

Synaptic shunting inhibition has been suggested as a rate control mechanism in the neocortex (Douglas et al., 1995). When a neuron is at rest, shunting inhibition does not produce a net hyperpolarizing current because its reversal potential $V_{I}$ is close to the resting potential. Instead, it causes an increase in membrane conductance, which divides the excitatory synaptic current (Carandini and Heeger, 1994). However, as it was recently pointed out by Holt and Koch (1997), the situation is different when the cell is in a repetitively firing state. In that case, the spiking mechanism essentially clamps the average membrane potential roughly half way between $V_{\text {reset }}$ and $V_{t h}$, well above $V_{I}$ (for example, $V_{I}=-70 \mathrm{mV}$, whereas $V_{a v}=\left(V_{\text {reset }}+V_{t h}\right) / 2=$ $-55.5 \mathrm{mV}$ ), and the effect of inhibitory synapses is hyperpolarizing. For example, suppose that the model network is in a persistently active state, and each neuron receives a feedforward synaptic inhibition with a given input rate $R_{I}$. Then, this input is equivalent to a negative current $I_{G A B A}=g_{G A B A} s_{I}\left(V_{a v}-V_{I}\right)$, where the synaptic drive $s_{I}$ as a function of $R_{I}$ is given by Equation 10. Therefore, the addition of feedforward inhibition simply shifts a frequency-current curve to the right by the fixed amount $I_{G A B A}$, without changing the range of network bistability or the lowest firing rate of a persistent state. This conclusion was confirmed by simulations (data not shown).

In the case of feedback synaptic inhibition, the firing of inhibitory interneurons is driven by pyramidal cells, and $R_{I}$ is a function of $R_{E}, R_{I}=g\left(R_{E}\right)$ (Fig. $7 C$ ). In this case $I_{b}$ remains the same, because $g_{G A B A}$ has no effect on the rest state. On the other hand, a larger $I$ is needed to counterbalance $I_{G A B A}$ for the persistent activity $\left(I_{G A B A}\right.$ shifts $I_{a}$ to the right). Therefore the range of network bistability $\left(I_{b}-I_{a}\right)$ is reduced. Note that, with increasing $g_{G A B A}$, although the firing rate at a given $I$ is reduced, the lowest possible rate of a persistent state (Fig. 7 A, filled square) remains almost the same. Therefore, recurrent inhibition acts in a subtractive manner, in the sense that is produces a negative
A

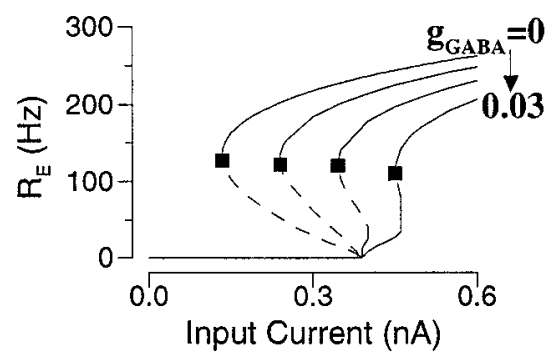

B
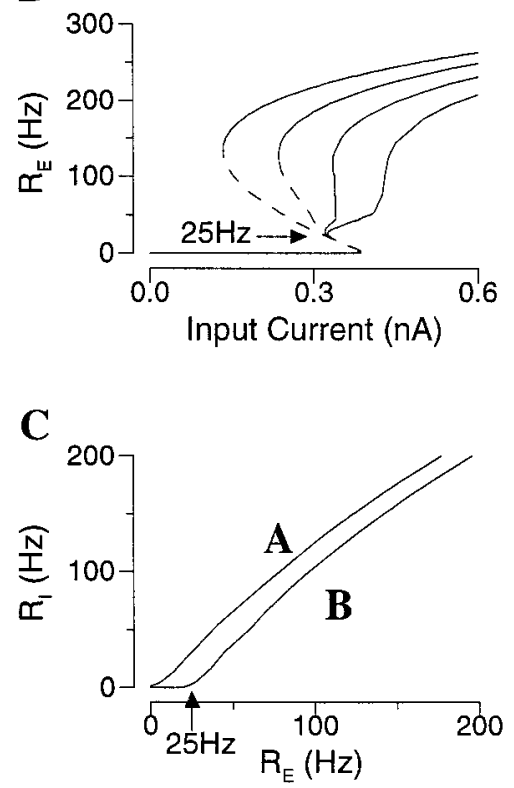

Figure 7. Effect of feedback shunting inhibition. $A, B$, Frequency-current curves with different $g_{G A B A}$ values when isolated interneurons are near or well below the firing threshold, respectively $(C)$. $A$, Stronger $g_{G A B A}$ reduces the bistable range and abolishes the persistent state. Note that the lowest firing rate of persistent activity ( filled square) is hardly changed by inhibition. $B$, In this case, the portion of the frequency-current curve with $R_{E}<25 \mathrm{~Hz}$ is unaffected by recurrent inhibition. With sufficiently large $g_{G A B A}$, bistability is preserved, and the active states have reasonably low firing rates $(25-50 \mathrm{~Hz}) . C$, The firing rate $R_{I}$ of interneurons as function of $R_{E}$ for $A$ and $B\left(g_{A M P A, e e}=1.2, g_{A M P A, e i}=0.4\right.$; the Poisson input rate to interneurons is $\lambda=2500 \mathrm{~Hz}$ in $A$ and $2000 \mathrm{~Hz}$ in $B$ ).

current that counterbalances the recurrent excitatory synaptic current. In terms of the firing rate equation $R_{E}=f\left(I_{t o t}\right)$, we have:

$$
I_{t o t}=I_{a p p}-I_{s y n, e e}-I_{s y n, i e}=I_{a p p}+\tilde{g}_{A M P A} R_{E}-\tilde{g}_{G A B A} s_{I}\left(R_{I}\right),
$$

with $\tilde{g}_{G A B A}=g_{G A B A}\left(V_{a v}-V_{I}\right)$ and $R_{I}=g\left(R_{E}\right)$ (Eq. 14). The subtractive term is nonlinear in $R_{E}$.

If inhibitory neurons are not near the firing threshold, they will fire spikes only when their excitatory drive is sufficiently strong, e.g. $R_{I}=0$ unless $R_{E}$ is above a critical value $\sim 25 \mathrm{~Hz}$ (Fig. $7 C$ ). As a result, the portion of the frequency-current curve of the pyramidal cell with $R_{E}<25 \mathrm{~Hz}$ (on the middle branch) cannot be altered by feedback inhibition. With sufficiently strong $g_{G A B A}$, network bistability is always preserved, and the lowest firing rate of a persistent state remains $25 \mathrm{~Hz}$ (Fig. $7 B$ ). In this way, persistent activity with reasonably low firing rates becomes possible.

I also considered an additional effect that may be caused by shunting inhibition. Suppose that shunting inhibition produces an 
increase in membrane conductance along a dendritic cable of length $L$, between the excitatory synapses and the spike triggering zone. The effective characteristic cable length $\lambda$ is then expected to decrease like $\lambda \sim\left(g_{L}+g_{G A B A} S_{I}\right)^{-1 / 2}$. To take into account the exponential attenuation of excitatory synaptic inputs along a passive cable, the excitatory conductance $g_{E}$ should be multiplied by a factor $\sim \exp (-L / \lambda) \sim \exp \left(-\beta\left(g_{G A B A S_{I}}\right)^{1 / 2}\right)$, where $\beta$ is given in terms of the cable properties (Abbott, 1991). This highly nonlinear effect was suggested to provide a solution to the high firing rate problem in neural networks (Abbott, 1991). When this effect is included in the model, persistent states with low firing rates can be obtained, the frequency-current curve of the pyramidal cell is similar to Figure $7 B$ (data not shown).

In any case, when a persistent state with low firing rate is realized with synaptic inhibition, its stability still remains to be determined. In fact, such a state was never observed in the network simulations, if the recurrent excitation was mediated exclusively by the fast AMPARs. Again, intuitively, such an active state is expected to be unstable because of the interplay between a fast recurrent excitation and a slower negative feedback. This is shown mathematically in the Appendix. To illustrate this point by computer simulations, I used the scaling parameter $\phi$ for the synaptic kinetics (Eqs. 4, 5) to change systematically the EPSC gating rates, whereas the average synaptic drive $s_{E}$ and the firing rate $R_{E}$ remained the same. $\phi$ was varied so that $\tau_{E}=\tau_{s} / \phi$ was between 2 and $80 \mathrm{msec}$. Let us choose $g_{G A B A}=0.03$ and $I=0.34$ $\mathrm{nA}$, the persistent state has a firing rate of $33 \mathrm{~Hz}$ (Fig. $7 B$ ). As shown in Figure 8, when the excitatory synapses are slow $\left(\tau_{E}=80\right.$ msec; comparable to that of the NMDAR channels), a persistent state can be sustained in the network (Fig. $8 A$ ). Because of the slow synaptic build-up, the network firing activity gradually ramps up during the input pulse. Moreover, in contrast to partially synchronous activity of Figure 4, with slow synaptic excitation (in the absence of a fast component) the persistent state is asynchronous. When $\tau_{E}$ is sufficiently reduced, the network activity in the persistent state displays increasingly large temporal fluctuations (Fig. $8 B$ ). If $\tau_{E}$ is decreased below a critical value $\left(\tau_{E} \simeq 18 \mathrm{msec}\right)$, the persistent state becomes unstable, because synchronous fluctuations eventually bring the network too close to the rest state, and the activity terminates (Fig. $8 \mathrm{C}$ ).

To conclude, the effect of $\mathrm{GABA}_{A}$ synaptic inhibition is largely subtractive rather than divisive in repetitively firing neurons. Therefore, the phenomenon of persistent activity becomes less robust and can be abolished completely by strong recurrent inhibition. Moreover, when a persistent state with low rate does exist, it cannot be stably maintained unless the excitatory synapses are sufficiently slow (the ratio $\tau_{E} / \tau_{I}$ must not be too small).

\section{Short-term synaptic depression}

I now turn to short-term depression of the excitatory synapses as a rate control mechanism. A typical simulation result is shown in Figure 9. In the absence of short-term depression (the parameter $p_{v}=0$; see Materials and Methods), a persistent activity state has a firing rate close to $200 \mathrm{~Hz}$ (Fig. 9A). The addition of short-term depression $\left(p_{v}=0.35\right)$ reduces the firing rate to $\sim 40 \mathrm{~Hz}$, back to the physiological range of PFC neurons (Fig. 9B). Note that, because of short-term depression, the neuronal firing shows an exponential decrease during the depolarizing input pulse (Fig. $9 B$, top and middle panels); and immediately after the pulse there is a trough in the neural activity during which time the synapses recover from depression (Fig. $9 B$, bottom panel). In this simula-
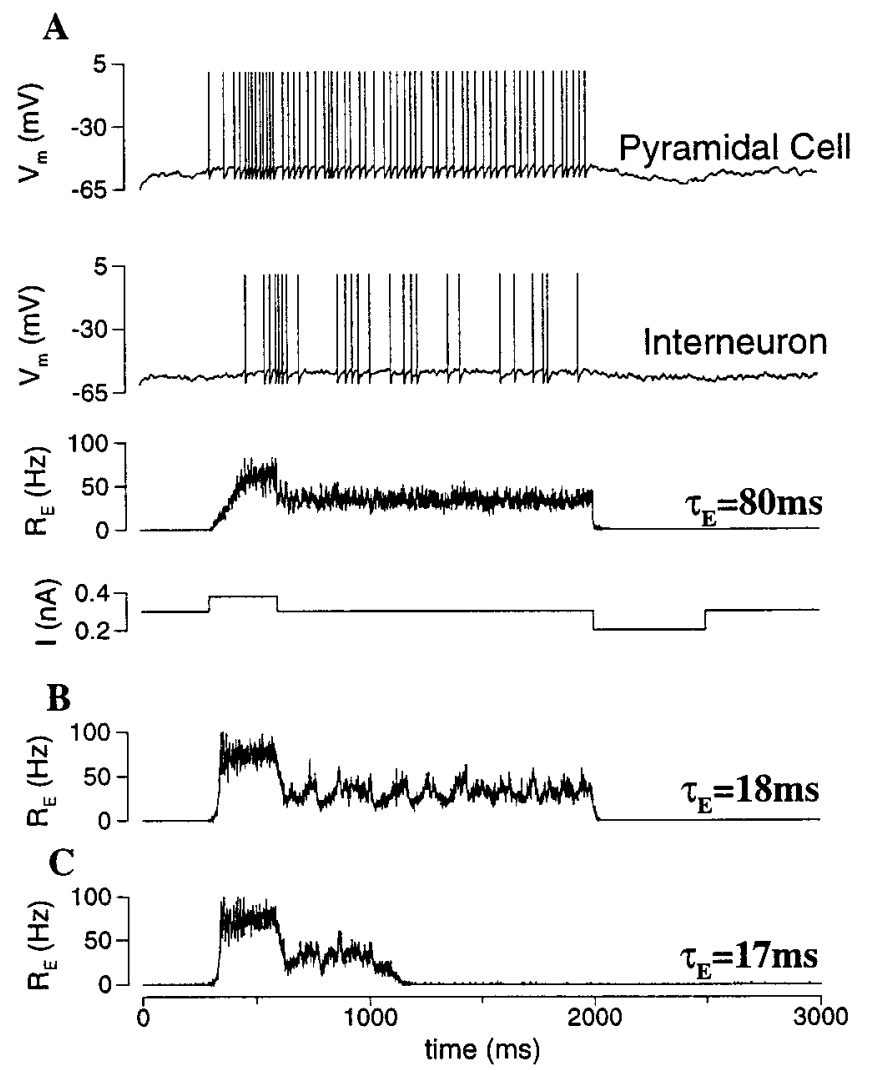

Figure 8. The low rate asynchronous state is not stable if excitatory synapses are too fast. The network model is simulated in the presence of strong recurrent inhibition. The speed of the excitatory synaptic kinetics is varied, whereas the steady-state synaptic drive and the mean firing rate are preserved. $A$, With $\tau_{E}=80 \mathrm{msec}$, the network can be turned on to the persistent state with $R_{E} \simeq 33 \mathrm{~Hz}$. Note the slow ramping-up of $R_{E}(t)$ during the transient stimulus, caused by the temporal summation of the slow synaptic current. $B$, With $\tau_{E}=18 \mathrm{msec}$, the persistent state is still stable, but $R_{E}(t)$ displays large fluctuations in time. $C$, With $\tau_{E}=17 \mathrm{msec}$, the fluctuations eventually bring $R_{E}(t)$ too close to zero, and the network returns to the rest state (same parameters as in Fig. $7 B$, with $g_{G A B A}=0.03$ and $I=0.34 \mathrm{nA})$.

tion both fast AMPAR and slow NMDAR channels are included, and the dynamics is asynchronous in the persistent state.

The frequency-current curve is calculated for different degrees of short-term depression (Fig. 10A). In this case the undepressed AMPAR-mediated currents are so strong that with $p_{v}=0$ the firing rates of the persistent states are $\sim 500 \mathrm{~Hz}$, near the neuronal saturation (data not shown). As we see in Figure $10 A$, shortterm depression dramatically decreases the lowest firing rate of the active states (Fig. $10 \mathrm{~A}$, filled square). The range of bistability also shrinks ( $I_{a}$ shifts to the right) with increasingly strong shortterm depression; but for some $p_{v}$ values this range remains reasonably large while the physiological firing rates are achieved. Short-term depression gives rise to synaptic saturation, which occurs at lower firing rates with larger $p_{v}$ (Fig. 10B). Indeed, for AMPAR channels $s_{E} \simeq \nu R_{E}$. With short-term depression $s_{E}=$ $\nu R_{E} /\left(1+p_{v} \tau_{D} R_{E}\right)$ (Eq. 9). In terms of the firing rate equation $R=f\left(I_{\text {tot }}\right)$, we have:

$$
I_{\text {tot }}=I_{\text {app }}-I_{\text {syn }}=I_{\text {app }}+\frac{\tilde{g}_{A M P A} R_{E}}{1+p_{v} \tau_{D} R_{E}} .
$$

Therefore, the effect of short-term depression divides the amplitude of the excitatory synaptic drive. Unlike a subtractive mech- 


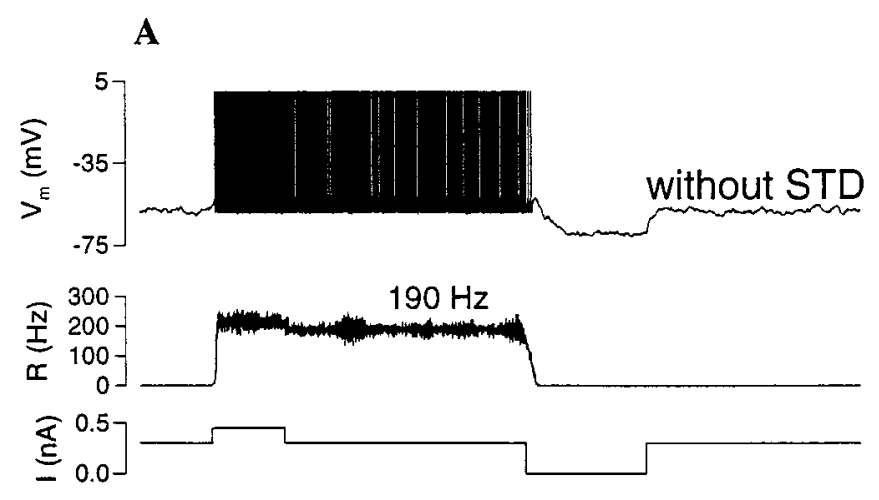

B
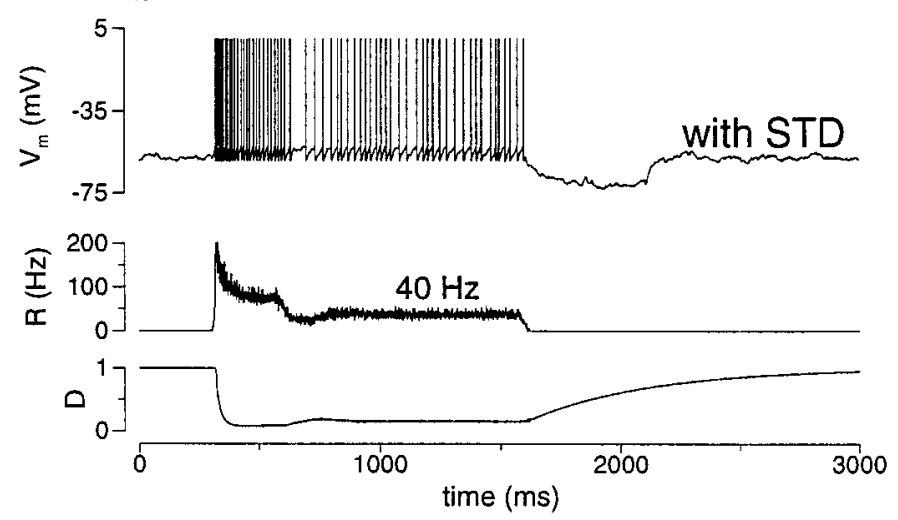

Figure 9. Rate control by short-term synaptic depression (STD). A, Without STD the firing rate of the persistent state is typically high, as long as there is a substantial AMPAR-mediated component of the recurrent synaptic transmission. $B$, The addition of STD $\left(p_{v}=0.3\right)$ significantly reduced the firing rate to $\sim 40 \mathrm{~Hz}$, within the physiological range of PFC cells. Note that during the transient depolarizing pulse $R(t)$ has a rapid peak, then decreases to a low steady state caused by STD (see $D(t))$. There is a trough in $R(t)$ immediately after the input pulse, when $D(t)$ recovers and reaches a steady state $\left(g_{A M P A}=0.7 ; g_{N M D A}=0.07 ; I=0.3 \mathrm{nA}\right)$.

anism (spike-frequency adaptation or recurrent inhibition), which is equally strong at all rates, a divisive mechanism affects high rates disproportionally. This leads to the flattening of the $f\left(I_{\text {tot }}(R)\right)$ curve (Fig. $\left.10 B\right)$. At high frequencies $\left[R_{E} \gg 1 /\left(p_{v} \tau_{D}\right)\right]$, the synaptic current becomes independent of the firing rate (Abbott et al., 1997). As a result, the positive feedback between firing and synaptic excitation has to stop at some firing rate, well below the neuronal saturation level $(\sim 500 \mathrm{~Hz})$.

The dynamical stability of these asynchronous persistent states with short-term depression was checked in direct network simulations. Simulations were performed with both all-to-all and sparse couplings. In a sparse network, unlike an all-to-all network, the number of synaptic connections varies widely from cell to cell, with an average $M_{s y n}$. One might expect that such heterogeneity would favor an asynchronous persistent state against instability and synchrony. In fact, I found that as long as $M_{s y n}$ is not too small $(\geq 100)$, the network behaves similarly with sparse or all-to-all coupling. This is true independent of the network size $N_{e}$. In other words, what matters is the absolute number of connections per neuron $M_{s y n}$, not the connection probability $p=M_{s y n} / N_{e}$. Similar to the case of spike-frequency adaptation or recurrent inhibition, it was found that fast AMPAR channels could not sustain such a low rate state, and that slower synapses were required (See Appendix for stability analysis). To be quantitative,
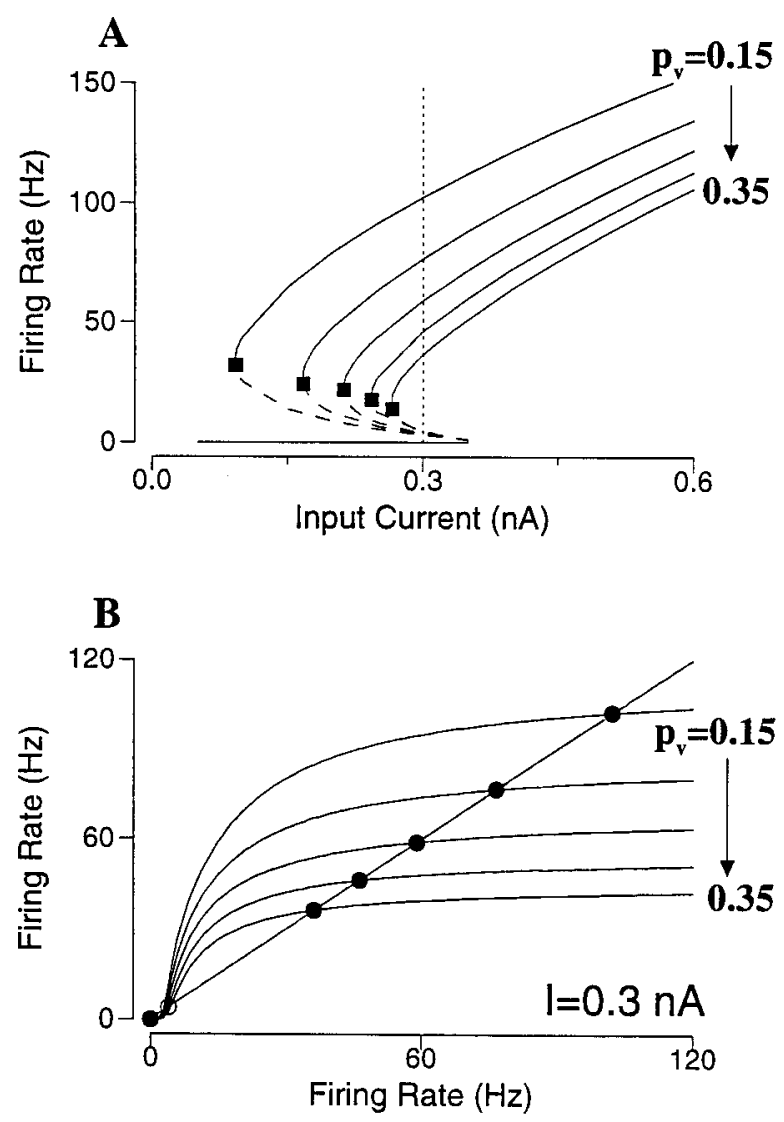

Figure 10. Effect of short-term synaptic depression in an excitatory neural network. $A$, Frequency-current curves with $p_{v}=0.15$ to 0.35 , by increment of 0.05 . Short-term depression reduces the lowest firing rates of the active states (filled square), whereas the bistable range remains reasonably large. $B$, For a fixed input current $(I=0.3 \mathrm{nA})$ in $A$, the firing rate of the asynchronous state is given by $R=f(R)$; or the intersections of $f(R)$ with the diagonal line. Stronger short-term depression leads to saturation of the function $f(R)$ at progressively lower firing rates, so that rate control is achieved for the persistent state. $\left(g_{A M P A}=8\right)$.

for a given persistent state I varied the synaptic time constants systematically in network simulations by changing the scaling parameter $\phi($ Eqs. 4,5$)$. This way, the smallest value of $\tau_{E}=\tau_{s} / \phi$ that was needed for the persistent state to be observable was determined. For example, consider the persistent states at $I=0.3$ nA of Figure $10 A$, which have the firing rate ranged from 100 to $35 \mathrm{~Hz}$ as $p_{v}$ is varied from 0.15 to 0.35 . The minimal $\tau_{E}$ required for the stability of each of these states is plotted as function of the firing rate $R$ in Figure $11 A$. The critical $\tau_{E}$ is larger with lower $R$, it also depends on the time constant of the depression process $\tau_{D}$ (see Appendix).

Figure $11 A$ was obtained with $M_{s y n}=100$ for a sparse network. The dependence on $M_{s y n}$ is shown in Figure $11 B$, for $R=35 \mathrm{~Hz}$. One observes that the minimal $\tau_{E}$ is not sensitive to $M_{s y n}$, as long as $M_{s y n} \geq 100$. At very small $M_{s y n}$, there is an abrupt increase of the required minimal $\tau_{E}$, i.e. even slower synapses are needed to stabilize the active state. This is because, if a neuron receives a very small number of synaptic inputs, each at a low rate, the synaptic current must be long-lasting in order to produce a sustained tonic drive to the postsynaptic cell. Figure 11, $C$ and $D$, illustrates the network dynamical behavior for $\tau_{E}$ around the critical minimum for $R=35 \mathrm{~Hz}\left(M_{\text {syn }}=100\right)$. In these simulations, the network was initially set to be very close to the asyn- 


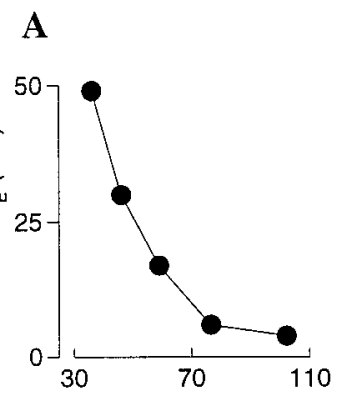

Rate of Persistent State $(\mathrm{Hz})$

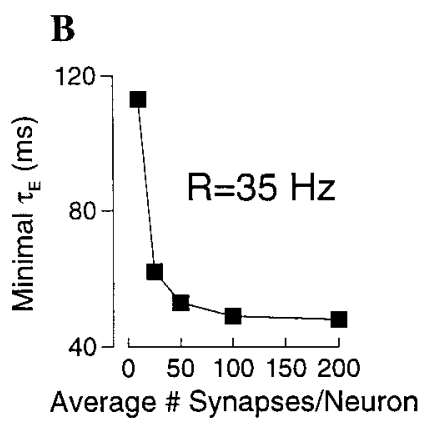

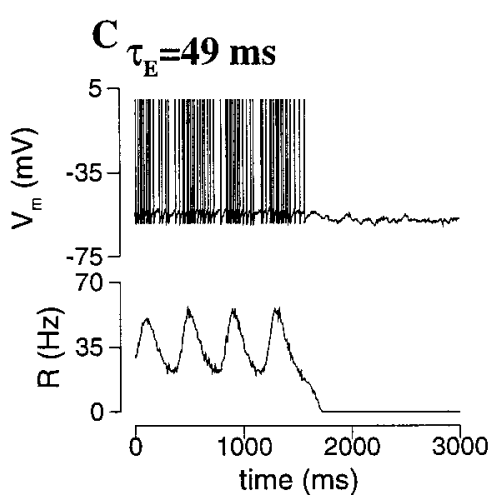
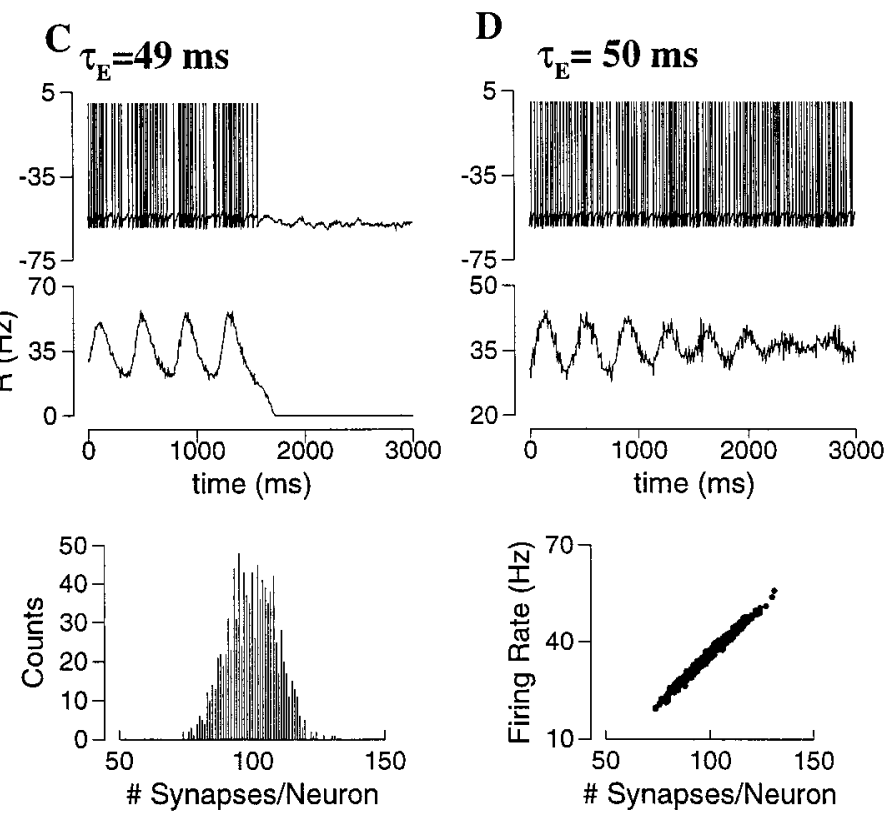

Figure 11. Stability of the persistent state in a sparse network with short-term depression (average number of synapses per neuron $M_{\text {syn }}=100$ except for $B$ ). $A$, For each of the five active states in Figure $10 B$, the network model is simulated, whereas the synaptic time constant $\tau_{E}$ is varied systematically. The minimal value of $\tau_{E}$ for which the persistent state was observed is plotted against the firing rate. Thus, the lower is the firing rate, the slower the synapses must be to sustain the network persistent activity. $B$, The required minimal $\tau_{E}$ is not sensitive to $M_{s y n}$, as long as the latter is $>100$. $C$, $D$, An example with $p_{v}=0.35$ and $R=35 \mathrm{~Hz}$. The initial condition for the network simulation was prescribed to be as close to the asynchronous state as possible. $C$, For $\tau_{E}=49 \mathrm{msec}$, the fluctuations of the network activity as measured by $R(t)$ grow in time, and eventually die out. Bottom panel, Histogram of the number of connections per neuron, centered at $M_{s y n}=100$. $C$, For $\tau_{E}=50 \mathrm{msec}$, network fluctuations are damped out, and the persistent state is stabilized. Bottom panel, The neural firing rate is a linear function of the number of synaptic inputs and varies in a wide range $(20-60 \mathrm{~Hz})$ across the population.

chronous state (see Materials and Methods for the asynchronous initial condition). Below the critical value (Fig. $11 C ; \tau_{E}=49$ msec), the synchronous state is unstable. The network activity fluctuates in time, and $R$ oscillates with growing amplitude. When $R$ gets close to zero, the synaptic excitation becomes too weak to bring the network back up again, and the network activity dies out (Fig. $11 C$ ). On the other hand, above the critical value (Fig. $11 D ; \tau_{E}=50 \mathrm{msec}$ ), fluctuations of the network activity decay with time, and the asynchronous persistent state is stable. In this random and sparse network, the firing rate of a neuron is a linear function of its number of synaptic connections (Fig. 11D, bottom panel), and is widely distributed across the neural population $(20-60 \mathrm{~Hz})$.

To conclude, unlike spike-frequency adaptation or synaptic inhibition, short-term depression acts as a divisive mechanism for rate control. The resulting persistent states at low firing rates are not stable, unless $\tau_{E}$ is larger than a critical value, which depends on both the short-term depression time constant and the firing rate. For the firing rates in the physiological range of PFC cells, the required synaptic kinetics is much slower than that of the AMPAR channels.

\section{DISCUSSION}

The general finding of this work is that memory processes performed in strongly recurrent cortical circuits, such as delay-period activity, depend on the temporal dynamics as much as on the efficacy of recurrent synapses. Three main conclusions are: (1) the asynchronous dynamics is generally not stable in a fast recurrent excitation/slow negative feedback system; (2) slow NMDARactivated synapses are powerful for maintaining a stable persis- tent activity at low firing rates; (3) short-term depression of excitatory synapses provides an efficient mechanism for rate control.

\section{NMDA receptors and persistent activity}

NMDAR channels were found to be crucial to persistent activity in the network model for two reasons. First, their slow gating kinetics naturally leads to synaptic saturation at low firing rates, as observed experimentally (Fig. $1 A$ ), thereby contributing to the rate control of network activity. This saturation of the steadystate response to repetitive stimulation should be distinguished from receptor saturation by a single vesicle of transmitter; the latter is not supported by recent data (Mainen et al., 1999) and is not assumed in the present model. Second, slow synapses usually suppress network instability and oscillations, but are also able to sustain a partially synchronized network dynamics realized by other (fast) mechanisms. The voltage dependence of gating kinetics represents another interesting feature of $I_{N M D A}$ to be explored in the context of working memory processes (Lisman et al., 1998).

Is there experimental evidence for a critical role of NMDARs in delay-period activity of the prefrontal cortex? Scherzer et al. (1998) reported a much higher expression of the NMDAR subunit mRNAs in the prefrontal cortex than in other cortical areas (such as primary visual cortex) of the human brain; which raises the interesting question of whether this regional difference could be correlated with the conspicuous occurrence of persistent activity in the association cortices in contrast to sensory cortices. NMDARs have been demonstrated to contribute to synaptic transmission at intracortical connections of sensory cortices 
(Thomson et al., 1985; Larson-Prior et al., 1991; ArmstrongJames et al., 1993; Thomson and Deuchars, 1995; Markram et al., 1997) and frontal cortex (Sutor and Hablitz, 1989; Hirsch and Crépel, 1990; Kang, 1995). In certain cortical area, this contribution may overwhelm that of AMPARs and dominate recurrent horizontal excitations (Fleidervish et al., 1998). More quantitative analysis of the NMDAR- and AMPAR-mediated synaptic currents in the PFC has been lacking, for both the monkey and the rodent. On the other hand, in behavioral experiments with rats performing a spatial delayed alternation task, systematical administration (Verma and Moghaddam, 1996) or microinjection into the prefrontal cortex (Romanides et al., 1999) of NMDAR antagonists impaired working memory. These observations are consistent with our hypothesized importance of NMDARs to working memory. A direct experimental test, however, will need to be done on behaving animals, by combining pharmacological manipulation of NMDARs with neuronal recordings from the prefrontal cortex.

Note that in a model of persistent activity in the gaze control system, Seung (1996) also suggested that slow synaptic transmission is of crucial importance, but for quite different reasons. That network model is only weakly nonlinear, and slow synapses are useful to prolong the lifetime of transient memory storage.

\section{Rate control and robustness of network bistability}

I have tested three candidate rate control mechanisms: spikefrequency adaptation, feedback inhibition, and synaptic shortterm depression. I argue that a rate control mechanism should be assessed based on its effect on the entire frequency-current curve of the network. A rate control mechanism is judged effective if it reduces the lowest firing rate of persistent activity down to a physiologically plausible range; and at the same time the network bistability should remain robust within a reasonable parameter range. By these criteria, it was found that both spike-frequency adaptation and feedback inhibition are not adequate. Both act in a subtractive way, in the sense that each produces a negative current that counterbalances the recurrent excitatory synaptic current (Eqs. 16,17), and they readily abolish the persistent activity phenomenon. A note of caution is warranted there, because this study used the simple LIF neuron model that does not take into account more complex features of cortical neurons, such as dendritic morphology or other ionic currents that may contribute to single neuron dynamics. In particular, it would be worth reexamining the issue of feedback inhibition in a more realistic situation where, for example, shunting inhibition is located near the soma of a neuron, spatially separated from the excitatory inputs at dendritic sites. Moreover, our conclusion on shunting inhibition follows from the required preservation of network bistability, hence it does not deny the importance of recurrent inhibition as a rate control mechanism in situations without persistent activity, such as sensory processes in the primary visual cortex (Douglas et al., 1995; Borg-Graham et al., 1998). Finally, synaptic inhibition is likely indispensible for the formation of memory fields of the PFC neurons (Goldman-Rakic, 1995; Camperi and Wang, 1998; Rao et al., 1999).

In contrast to spike-frequency adaptation or synaptic inhibition, short-term synaptic depression acts as a divisive mechanism, in the sense that it divides the recurrent synaptic conductance (Eq. 18). Short-term depression reduces the firing rate not by preventing the neuronal saturation, but by saturating the synaptic drive at low firing rates (Fig. 10B). In the divisive but not subtractive case, firing rate of persistent activity is reduced effec- tively, whereas bistability is preserved in a robust way. Recent in vitro experiments have indicated that short-term depression is a general property of the rat PFC synapses (Hempel, Hartman, Wang, Turrigiano, and Nelson, unpublished observations). It would be interesting to see whether there is evidence for shortterm depression in firing patterns of PFC cells of the behaving animal. Similar to our model simulation (Fig. 9B), in a delayedresponse task, PFC neurons often display an exponential decrease of the firing rate during the cue presentation, followed by a trough of activity (Chafee and Goldman-Rakic, 1998; Romo et al., 1999; G. Rainer and E. K. Miller, personal communication). Such an effect needs to be measured quantitatively, and its underlying cellular mechanism remains to be elucidated.

\section{Stability and synchronization}

To sustain persistent activity, a tonic synaptic drive is required to remain significantly above zero at any moment. This can be achieved by the fast AMPA-type synapses alone, if neuronal firings are asynchronous. However, previous work has shown that the asynchronous state is dynamically unstable if the excitatory synapses are too fast (Abbott and van Vreeswijk, 1993). I found that this problem is much more serious in the presence of a strong negative feedback mechanism for rate control. A pertinent question is to what extent this conclusion holds true in the presence of additional factors that increase the disorder of the network. Previous work has shown that noise has a stabilizing effect on the asynchronous dynamics of a network of excitatory neurons (Abbott and van Vreeswijk, 1993; Gerstner, 1999). In another study, a random network of excitatory and inhibitory neurons, coupled with instantaneous synapses, was found to be less synchronous with sparser connectivity (Brunel, 1999). None of these models contains a slow negative feedback mechanism. Here, in a network where recurrent excitation interacts with slow short-term depression, I found that asynchronous dynamics is not stable if the excitatory synapses are fast, even in the presence of synaptic noise and when the network connectivity is very sparse and the neuronal firing properties are widely heterogeneous (Fig. 11). Further analysis is needed to see if asynchronous dynamics are generally unstable in such fast recurrent excitation/slow negative feedback systems, even in the presence of heterogeneity and noise. The problem of stability of the asynchronous dynamics is of interest in the larger context of balanced excitatory-inhibitory neural networks (Shadlen and Newsome, 1994; van Vreeswijk and Sompolinski, 1996).

Therefore, a general finding here is that when an asynchronous persistent state has a low firing rate, its stability requires that the excitatory synaptic time constant be comparable to the effective time constant of the negative feedback mechanism. For a recurrent network of pyramidal cells and interneurons, the stability of a persistent state critically depends on whether the $\mathrm{GABA}_{\mathrm{A}} \mathrm{R}$ mediated inhibition is as fast as the AMPAR-activated excitation. For both AMPARs (Geiger et al., 1995) and GABA Rs (Macdonald and Olsen, 1994), the deactivation kinetics is regulated by the subunit composition and thus may be specific for each cell type. In hippocampal pyramidal neurons of the rat, the decay time constant of the AMPAR-mediated EPSCs is $\simeq 2 \mathrm{msec}\left(\right.$ at $35^{\circ} \mathrm{C}$ ) (Hestrin et al., 1990a), whereas that of the fast component of the $\mathrm{GABA}_{\mathrm{A}} \mathrm{R}$-mediated IPSCs is $\simeq 6-10 \mathrm{msec}$ (Banks et al., 1998). Hence IPSCs are approximately three to five times slower than EPSCs. The present study showed that such a mismatch of synaptic time constants does not favor the stability of an asynchronous dynamics at low firing rates, and for this reason the slow 
NMDAR channels could be required for the maintenance of a persistent state.

It is an open question whether completely asynchronous dynamics is indeed the modus operandi of delay-period activity in the PFC circuit. Funahashi (1998) recently reported that simultaneously recorded PFC cells displayed significant temporal correlations in a spatial working memory task. In my model simulations, when both the fast AMPA and slow NMDAR-mediated synaptic components are present, the fast AMPAR-activated recurrent excitation in interplay with slower negative feedback processes often leads to synchronous neural firings and network oscillations. In such a synchronous persistent state, the decay time constant of the slow synaptic component must not be too small compared to the average interspike interval (or oscillation period) of neurons. For typical firing rates of PFC cells of $10-50 \mathrm{~Hz}$, ISI $\simeq 20-100 \mathrm{msec}$, the NMDAR channels are needed.

\section{From cellular physiology to behavior}

The present study raised and highlighted a number of experimental questions, their answers will contribute to bridge the gap between behavior-related neural activity and its underlying biological mechanisms.

\section{Synaptic physiology of the prefrontal cortex}

(1) What are the precise time courses of the AMPAR-mediated EPSCs and $\mathrm{GABA}_{\mathrm{A}} \mathrm{R}$-mediated IPSCs? Is there a mismatch between the two? (2) In response to a repetitive train of stimuli, is the NMDAR-mediated EPSC a linear function of the stimulus frequency in the steady state? If not, what is the frequency above which the current saturates? (3) What are the relative amplitudes of the AMPAR- and NMDAR-mediated EPSCs? Can they be differentially modulated by neuromodulators such as dopamine (Cepeda et al., 1992)? (4) Across the somatodendritic membrane of a pyramidal neuron, is there a spatial segregation of excitatory and inhibitory synapses? (5) What are the short-term plasticity properties of PFC synapses?

\section{Neural delay-period activity of the behaving animal}

(6) Is there evidence for adaptation/depression of neuronal discharges? (7) How variable/random is the neuronal persistent activity? Do spike trains display some regular temporal structure? (8) Do neurons fire asynchronously, or is there synchronization within neural assemblies? (9) Can persistent activity of a neuron be switched off by an intracellularly injected current pulse? (no, if delay-period activity is network-induced; yes, if there is bistability at the single cell level) (10) Would local blockade of NMDA receptors in the PFC impair an animal's working memory performance? What are the correlated changes in the delay-period activity of PFC neurons?

\section{Implications for schizophrenia}

In recent years, there is growing evidence that working memory impairments are prominent symptoms in schizophrenia (Goldman-Rakic, 1994; Weinberger and Berman, 1996), and that dysfunction of the NMDAR-mediated neurotransmission in the cortex may be at the origin of these cognitive deficits (Javitt and Zukin, 1991; Coyle, 1996). For example, a noncompetitive NMDA antagonist such as phencyclidine or ketamine produces working memory deficits in healthy human subjects that closely resemble schizophrenia (Javitt and Zukin, 1991; Krystal et al., 1994). Moreover, significant alternations in gene expression of the NMDA receptor subunits were found in PFC of schizophrenics (Akbarian et al., 1996). However, the cellular mechanisms through which working memory relies on the NMDAR channels are largely unknown. The present theoretical work suggests a candidate scenario for the working memory malfunction in PFC, namely, an imbalance between the fast AMPAR- and the slow NMDAR-mediated components of the recurrent synaptic transmission within the PFC circuit can give rise to network dynamical instability and disruption of delay-period persistent activity.

\section{APPENDIX}

\section{Stability of an asynchronous state}

In this Appendix, I show that, in general, an asynchronous persistent state is not stable in a fast recurrent excitation/slow negative feedback system. Using a heuristic approach, I will write a dynamical equation for the population activity, in each of the three cases: spike-frequency adaptation, synaptic shunting inhibition, and short-term synaptic depression. Then I will discuss in detail the stability analysis of such a dynamical system.

\section{General remark}

Because the excitatory network has a large number of dynamical variables (at least as many as the number of pyramidal cells), a rigorous stability analysis of the network involves as many degrees of freedom (Abbott and van Vreeswijk, 1993; Treves, 1993; Gerstner, 1999). However, our approach is to focus on the fastest and most stable of all dynamical modes for the system (when decoupled from the negative feedback). This would yield a single dynamical equation for the population firing rate which, combined with another equation describing the negative feedback, forms a two-variable system. The idea is that if a steady state is not stable for the two-variable system, it must be unstable for the original network. On the other hand, if it is stable by this description, it still is not necessarily stable for the full network system.

\section{Spike-frequency adaptation}

The starting point is the firing rate equation $R=f\left(R,\left[\mathrm{Ca}^{2+}\right]_{a v}\right) \cdot f$ is the neuronal input-output relation, and the input current includes contributions from the recurrent excitatory synaptic current, which itself depends on $R$, the adaptation current that is proportional to $\left[\mathrm{Ca}^{2+}\right]$, as well as the external current $I$ (not explicitly shown). Suppose that one can write a dynamical equation for $R$, like:

$$
\frac{d R}{d t}=F\left(R,\left[C a^{2+}\right]_{a v}\right)=\left(f\left(R,\left[C a^{2+}\right]_{a v}\right)-R / \tau_{E},\right.
$$

where $\tau_{E}$ is a characteristic time constant for the most stable dynamical mode of the network (when $g_{A H P}=0$ ). It is assumed to be dominated by the time constant of the excitatory synapses rather than the membrane time constant (Abbott and van Vreeswijk, 1993; Treves, 1993; Gerstner, 1999). The steady state is given by $d R / d t=0$, from which $R=f\left(R,\left[\mathrm{Ca}^{2+}\right]_{a v}\right)$ is recovered.

The equation for the calcium concentration averaged over a typical interspike interval $1 / R$ is:

$$
\frac{d\left[\mathrm{Ca}^{2+}\right]_{a v}}{d t}=G\left(R,\left[\mathrm{Ca}^{2+}\right]_{a v}\right)=\alpha_{C a} R-\left[\mathrm{Ca}^{2+}\right]_{a v} / \tau_{C a}
$$

Equations 19 and 20 constitute a dynamical system of two variables; it can be analyzed by the phase-plane technique (Strogatz, 1994; Rinzel and Ermentrout, 1998). It is convenient to choose $R$ and $g_{A H P}\left[\mathrm{Ca}^{2+}\right]$ as independent variables, so that the function $f\left(R, g_{A H P}\left[\mathrm{Ca}^{2+}\right]_{a v}\right)$ is the same for different adaptation 


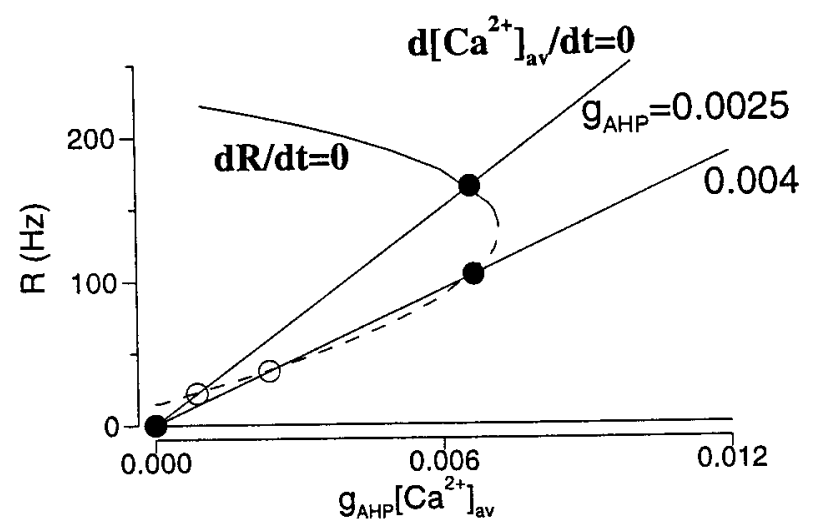

Figure 12. Phase-plane analysis for spike-frequency adaptation. The $R$-nullcline plotted as function of $g_{A H P}\left[\mathrm{Ca}^{2+}\right]$ is independent of $g_{A H P}$. The $\left[\mathrm{Ca}^{2+}\right]$-nullcline is given by Equation 20 . It is a straight line, with a decreasing slope for larger $g_{A H P}$. The steady states are given by the intersections of the two nullclines. The persistent state is located at the top branch of the $R$-nullcline with small $g_{A H P}$, and is moved to the middle branch with large $g_{A H P}$. This active state on the middle branch is not stable if the excitatory synaptic decay is much faster than the adaptation time constant. Same parameters as in Figure $6 A(I=0.35 \mathrm{nA})$.

strengths $g_{A H P}$. The nullcline $d R / d t=F\left(R,\left[\mathrm{Ca}^{2+}\right]_{a v}\right)=0$ plotted on the phase plane has three branches (Fig. 12). The first one is $R=0$. The second one is a decreasing function of $\left[\mathrm{Ca}^{2+}\right]_{a v}$, signifying a reduction of $R$ by adaptation. The third one is the middle branch connecting the other two branches. On the other hand, the nullcline $d\left[\mathrm{Ca}^{2+}\right]_{a v} d t=G\left(R,\left[\mathrm{Ca}^{2+}\right]_{a v}\right)=0$ is a straight line (Eq. 20). A steady state is given by an intersection of the two nullclines. For small $g_{A H P}$, there are three intersection points, one on each of the three branches. The third intersection point with the highest firing rate corresponds to the persistent state. With increasingly larger $g_{A H P}$, the $R$-nullcline remains the same (because it only depends on the product $\left.\mathrm{g}_{A H P}\left[\mathrm{Ca}^{2+}\right]_{a v}\right)$, whereas the $\left[\mathrm{Ca}^{2+}\right]_{a v}$ nullcline has a decreasing slope. With sufficiently large $g_{A H P}$, the persistent steady state is moved from the top branch to the middle branch of the $R$-nullcline (Fig. 12).

\section{Recurrent shunting inhibition}

In the case of shunting inhibition, we can heuristically write two coupled equations for the firing rates $R_{E}$ and $R_{I}$ of the excitatory and inhibitory neurons,

$$
\begin{gathered}
\frac{d R_{E}}{d t}=F\left(R_{E}, R_{I}\right)=\left(f\left(R_{E, R_{I}}\right)-R_{E}\right) / \tau_{E} \\
\frac{d R_{I}}{d t}=G\left(R_{E}, R_{I}\right)=\left(g\left(R_{E}\right)-R_{I}\right) / \tau_{I} .
\end{gathered}
$$

where $\tau_{E}$ and $\tau_{I}$ are the excitatory and inhibitory synaptic time constants, respectively. The steady states are given by $d R_{E} / d t=0$ and $d R_{I} / d t=0$, from which the neuronal input-output relations (Eq. 14) are recovered. Two examples with different inhibition strengths $\left(g_{G A B A}\right)$ are shown in Fig. 13, using the same parameters as in Figure $7 B$. As we see in Figure $13 A$, the $R_{E}$-nullcline has three branches, and the asynchronous active state $\left(R_{E}^{*}, R_{I}^{*}\right)$ with a high rate is located on the top one. When the firing rate $R_{E}$ is reduced sufficiently by strong recurrent inhibition, $\left(R_{E}^{*}, R_{I}^{*}\right)$ is moved to the middle branch of the $R_{E}$-nullcline (Fig. 13B).
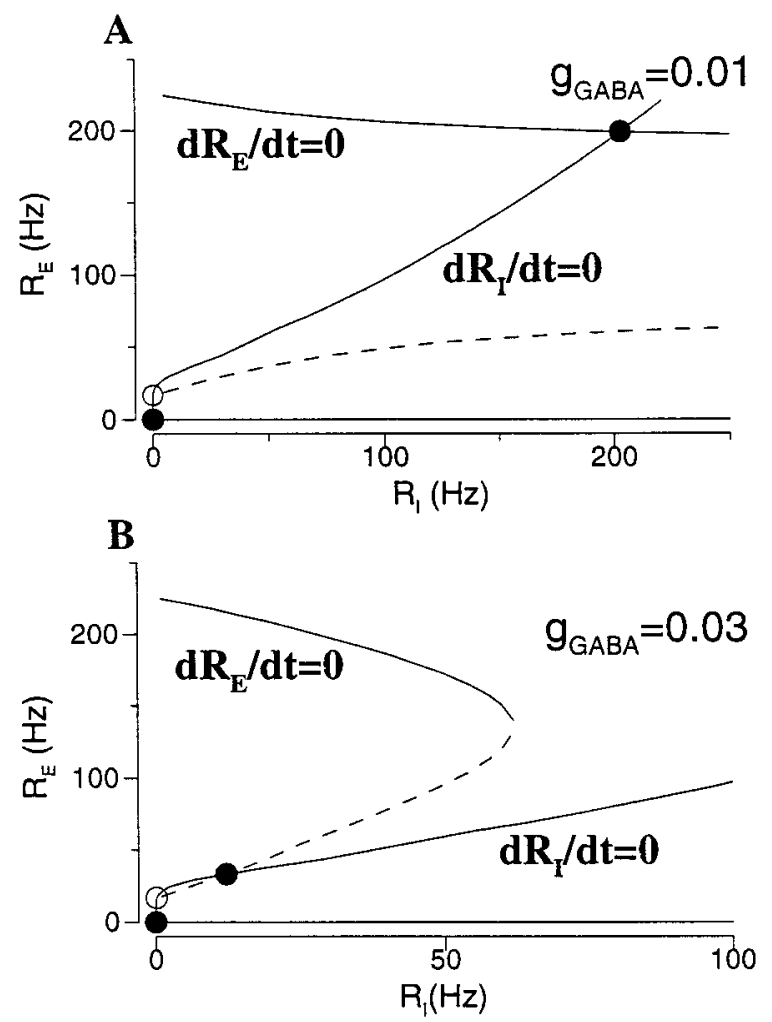

Figure 13. Phase-plane analysis for recurrent shunting inhibition. $A$, For small $g_{G A B A}$, the active state is located on the top branch of the $R_{E^{-}}$ nullcline. $B$, For large $g_{G A B A}$, it is shifted to the middle branch of the $R_{E}$-nullcline with a low firing rate. This active state on the middle branch is not stable if the excitatory synapse is fast compared to the inhibitory synapse. Same parameters as in Figure $7 B(I=0.34 \mathrm{nA})$.

\section{Short-term synaptic depression}

In this case, the equations are:

$$
\begin{gathered}
\frac{d R}{d t}=F(R, D)=(f(R, D)-R) / \tau_{E} ; \\
\frac{d D}{d t}=G(R, D)=-p_{v} R D+(1-D) / \tau_{D},
\end{gathered}
$$

the steady states are given by $d R / d t=0$ and $d D / d t=0$, which yield $R=f(R, D)$ and $D=1 /\left(1+p_{v} R \tau_{D}\right)$. These nullclines are plotted in Fig. 14, for two different $p_{v}$ values. Again, the $R$-nullcline has three branches. The asynchronous persistent state with a high firing rate is on the top branch, and is moved to the middle branch when its rate is reduced by strong synaptic depression.

\section{Stability analysis}

We have seen that in each of the three cases, the $R_{E}$-nullcline has three branches (the usual situation when there is a bistability between the rest state and an active state). The asynchronous active state, whose rate is reduced into the physiological range by a negative feedback mechanism, is typically located on the middle branch of the $R$-nullcline. I would like to show that this persistent state is not stable if the excitatory synapses are much faster than the effective time constant of the negative feedback. I will consider only the case of recurrent synaptic inhibition. The other two cases can be treated in a similar manner.

To study the local stability of a persistent state $\left(R_{E}^{*}, R_{I}^{*}\right)$, in the 


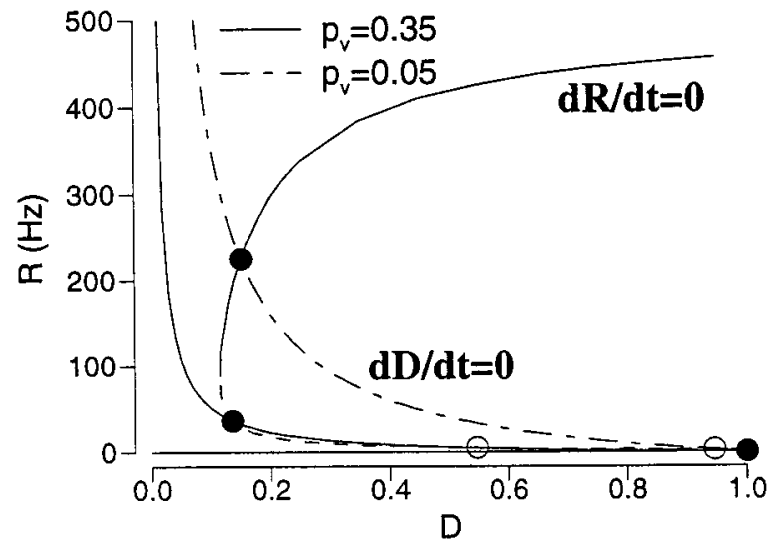

Figure 14. Phase-plane analysis for short-term synaptic depression. The $R$-nullcline is independent of the depression parameter $p_{v}$, and the $D$-nullcline is shown with two $p_{v}$ values. For small $p_{v}$, the persistent state is located on the top branch of the $R$-nullcline. For large $p_{v}$, it is shifted to the middle branch with reduced firing rate. This active state is not stable if the excitatory synaptic decay is much faster than the effective time constant of synaptic depression. Same parameters as in Figure $10(I=0.3 \mathrm{nA})$.

presence of shunting inhibition, we shall linearize Equations 21 and 22. The local stability is determined by the matrix:

$$
M=\left(\begin{array}{cc}
\frac{\partial F}{\partial R_{E}} \frac{\partial F}{\partial R_{I}} \\
\frac{\partial G}{\partial R_{E}} \frac{\partial G}{\partial R_{I}}
\end{array}\right)=\left(\begin{array}{cc}
\left(\frac{\partial f}{\partial R_{E}}-1\right) / \tau_{E} \frac{\partial f}{\partial R_{I}} / \tau_{E} \\
\frac{\partial g}{\partial R_{E}} / \tau_{I} & -1 / \tau_{I}
\end{array}\right),
$$

evaluated at $\left(R_{E}^{*}, R_{I}^{*}\right)$. This steady state is locally stable, if the two eigenvalues $\lambda_{1}$ and $\lambda_{2}$ of $M$ have a negative real part. A qualitative analysis can be performed as follows. Let us define $\operatorname{Tr}=$ $\partial F / \partial R_{E}+\partial G / \partial R_{I}$, and Det $=\left(\partial F / \partial R_{E}\right)\left(\partial G / \partial R_{I}\right)-\left(\partial F / \partial R_{I}\right)$ $\left(\partial G / \partial R_{E}\right)$, then $\lambda_{1}$ and $\lambda_{2}$ are solutions of the algebraic equation $\lambda^{2}-\operatorname{Tr} \lambda+$ Det $=0$; and $\lambda_{1}+\lambda_{2}=\operatorname{Tr}, \lambda_{1} \lambda_{2}=$ Det.

Because $F$ decreases with $R_{I}$ and $G$ increases with $R_{E}$, we have:

$$
\frac{\partial F}{\partial R_{I}}<0 ; \quad \frac{\partial G}{\partial R_{E}}>0, \quad \frac{\partial G}{\partial R_{I}}=-1 / \tau_{I}<0,
$$

and the sign of $\partial F / \partial R_{E}$ depends on which branch of the $R_{E^{-}}$ nullcline is the steady state located. Moreover, we shall make use of the information about the slope of each nullcline at $\left(R_{E}^{*}, R_{I}^{*}\right)$ (Fig. 13). For the $R_{E}$-nullcline,

$$
\frac{\partial F}{\partial R_{E}} d R_{E}+\frac{\partial F}{\partial R_{I}} d R_{I}=0 ; \quad \text { or }\left(\frac{d R_{E}}{d R_{I}}\right) R_{E}=-\left(\frac{\partial F}{\partial R_{I}}\right) /\left(\frac{\partial F}{\partial R_{E}}\right),
$$

and for the $R_{I}$-nullcline,

$$
\frac{\partial G}{\partial R_{E}} d R_{E}+\frac{\partial G}{\partial R_{I}} d R_{I}=0 ; \quad \text { or }\left(\frac{d R_{E}}{d R_{I}}\right) R_{I}=-\left(\frac{\partial G}{\partial R_{I}}\right) /\left(\frac{\partial G}{\partial R_{E}}\right) .
$$

Suppose first that $\left(R_{E}^{*}, R_{I}^{*}\right)$ is on the top branch (Fig. 13A), where the slope of the $R_{E}$-nullcline is negative, $\left(\frac{\partial R_{E}}{\partial R_{I}}\right) R_{E}<0$. Combining Equation 27 with Equation 26, we have:

$$
\partial F / \partial R_{E}<0 .
$$

From Equations 26 and 29, we deduce that $\lambda_{1}+\lambda_{2}=\operatorname{Tr}<0$ and $\lambda_{1} \lambda_{2}=$ Det $>0$. If $\lambda_{1}$ and $\lambda_{2}$ are real, clearly they must both be negative, because their sum is negative, and their product is positive. If $\lambda_{1}$ and $\lambda_{2}$ are complex, their real part is $\operatorname{Tr} / 2<0$. In both cases, we conclude that the steady state is stable.

Let us now assume that the steady state $\left(R_{E}^{*}, R_{I}^{*}\right)$ is on the middle branch (Fig. $13 B$ ), where the slope of the $R_{E}$-nullcline is positive, $\left(\frac{d R_{E}}{d R_{I}}\right) R_{\mathrm{E}}>0$. Thus,

$$
\partial F / \partial R_{E}>0 .
$$

Because the slope of the $R_{E}$-nullcline is larger than that of the $R_{I}$-nullcline at $\left(R_{E}^{*}, R_{I}^{*}\right)$, we have:

$$
-\left(\frac{\partial G}{\partial R_{I}}\right) /\left(\frac{\partial G}{\partial R_{E}}\right)<-\left(\frac{\partial F}{\partial R_{I}}\right) /\left(\frac{\partial F}{\partial R_{E}}\right) .
$$

This, combined with Equations 26 and 30, leads to Det $=\lambda_{1} \lambda_{2}>0$.

Note that $\partial F / \partial R_{E}(>0)$ and $\partial G / \partial R_{I}(<0)$ are proportional to $1 / \tau_{E}$ and $1 / \tau_{I}$; respectively. Therefore, the sign of $T r=\partial F / \partial R_{E}+$ $\partial G / \partial R_{I}$ depends on the relative speeds of recurrent excitation and feedback inhibition. Suppose that $\tau_{E}$ is much smaller than $\tau_{I}$, the positive term dominates and $\operatorname{Tr}=\lambda_{1}+\lambda_{2}>0$. Because $\lambda_{1} \lambda_{2}>$ 0 , if $\lambda_{1}$ and $\lambda_{2}$ are real, then they must be positive; if they are complex, then their real part is positive. In both cases, the asynchronous active state is unstable. If $\tau_{E}$ is much larger than $\tau_{I}$, the negative term dominates, thus $\operatorname{Tr}=\lambda_{1}+\lambda_{2}<0$. Together with $\lambda_{1} \lambda_{2}>0$, we conclude that the asynchronous active state is stable within this framework of the population-activity description.

\section{REFERENCES}

Abbott LF (1991) Realistic synaptic inputs for model neural networks. Network 2:245-258.

Abbott LF, van Vreeswijk C (1993) Asynchronous states in networks of pulse-coupled oscillators. Phys Rev 48:1483-1490.

Abbott LF, Varela JA, Sen K, Nelson SB (1997) Synaptic depression and cortical gain control. Science 275:220-224.

Akbarian S, Sucher NJ, Bradley D, Tafazzoli A, Trinh D, Hetrick WP, Potkin SG, Sandman CA, Bunney Jr WE, Jones EG (1996) Selective alterations in gene expression for NMDA receptor subunits in prefrontal cortex of schizophrenics. J Neurosci 16:19-30.

Amari S (1977) Dynamics of pattern formation in lateral-inhibition type neural fields. Biol Cybern 27:77-87.

Amit DJ (1995) The hebbian paradigm reintegrated: local reverberations as internal representation. Behav Brain Sci 18:617-626.

Amit DJ, Brunel N (1997) Model of global spontaneous activity and local structured activity during delay periods in the cerebral cortex. Cereb Cortex 7:237-252.

Amit DJ, Tsodyks MV (1991) Quantitative study of attractor neural networks retrieving at low spike rates. Network 2:259-294.

Amit DJ, Evans MR, Abeles M (1990) Attractor neural networks with biological probe records. Network 1:381-405.

Amit DJ, Brunel N, Tsodyks MV (1994) Correlations of cortical Hebbian reverberations: experiment versus theory. J Neurosci 14:6435-6445.

Armstrong-James M, Welker E, Callahan CA (1993) The contribution of NMDA and non-NMDA receptors to fast and slow transmission of sensory information in the rat S1 barrel cortex. J Neurosci 13:2149-2160.

Baddeley A (1986) Working memory. Oxford: Clarendon.

Banks MI, Li T-B, Pearce RA (1998) The synaptic basis of GABA A,slow. $_{\text {. }}$ J Neurosci 18:1305-1317.

Borg-Graham L, Monier C, Frégnac Y (1998) Visual input evokes transient and strong shunting inhibition in visual cortical neurons. Nature 393:369-373.

Brunel N (1999) Dynamics of sparsely connected networks of excitatory and inhibitory neurons. J Comput Neurosci, in press.

Camperi M, Wang X-J (1998) A model of visuospatial working memory in prefrontal cortex: recurrent network and cellular bistability. J Comput Neurosci 5:383-405.

Carandini M, Heeger DJ (1994) Summation and division by neurons in primate visual cortex. Science 264:1333-1336. 
Cepeda C, Radisavljevic Z, Peacock W, Levine MS (1992) Differential modulation by dopamine of responses evoked by excitatory amino acids in human cortex. Synapse 11:330-341.

Chafee MV, Goldman-Rakic PS (1998) Neuronal activity in macaque prefrontal area $8 \mathrm{a}$ and posterior parietal area 7ip related to memory guided saccades. J Neurophysiol 79:2919-2940.

Coyle JT (1996) The glutamatergic dysfunction hypothesis for schizophrenia. Harv Rev Psychiatry 3:241-253.

Destexhe A, Mainen Z, Sejnowski TJ (1994) Synthesis of models for excitable membranes, synaptic transmission and neuromodulation using a common kinetic framework. J Comput Neurosci 1:195-230.

Douglas RJ, Koch C, Mahowald M, Martin KAC, Suarez HH (1995) Recurrent excitation in neocortical circuits. Science 269:981-985.

Ermentrout GB (1994) Reduction of conductance-based models with slow synapses to neural nets. Neural Comput 6:679-695.

Fleidervish IA, Binshtok AM, Gutnick MJ (1998) Functionally distinct NMDA receptors mediate horizontal connectivity within layer 4 of mouse barrel cortex. Neuron 21:1055-1065.

Funahashi S (1998) Dynamic changes in interactions among prefrontal neurons in relation to working memory processes. Soc Neurosci Abstr 24:1426.

Funahashi S, Bruce CJ, Goldman-Rakic PS (1989) Mnemonic coding of visual space in the monkey's dorsolateral prefrontal cortex. J Neurophysiol 61:331-349.

Fuster J (1988) The prefrontal cortex, Ed 2. New York: Raven.

Fuster J, Alexander G (1971) Neuron activity related to short-term memory. Science 173:652-654.

Geiger JRP, Melcher T, Koh D-S, Sakmann B, Seeburg PH, Jonas P, Monyer H (1995) Relative abundance of subunit mRNAs determines gating and $\mathrm{Ca}^{2+}$ permeability of AMPA receptors in principal neurons and interneurons in rat CNS. Neuron 15:193-204.

Gerstner W (1999) Population of spiking neurons. In: Pulsed neural networks (Maass W, and Bishop CM, eds), pp 261-295. Cambridge, MA: MIT

Gnadt JW, Andersen RA (1988) Memory related motor planning activity in posterior parietal cortex of macaque. Exp Brain Res 70:216-220.

Goldman-Rakic PS (1994) Working memory dysfunction in schizophrenia. J Neuropsychiatry Clin Neurosci 6:348-357.

Goldman-Rakic PS (1995) Cellular basis of working memory. Neuron 14:477-485.

Hansel D, Mato G, Meunier C, Neltner L (1998) On numerical simulations of integrate-and-fire neural networks. Neural Comput $10: 467-484$.

Hebb DO (1949) Organization of behavior. New York: Wiley.

Helmchen F, Imoto K, Sakmann B (1996) $\mathrm{Ca}^{2+}$ buffering and action potential-evoked $\mathrm{Ca}^{2+}$ signaling in dendrites of pyramidal neurons. Biophys J 70:1069-1081.

Hestrin S, Sah P, Nicoll RA (1990a) Mechanisms generating the time course of dual component excitatory synaptic currents recorded in hippocampal slices. Neuron 5:247-253.

Hestrin S, Perkel DJ, Sah P, Manabe T, Renner P, Nicoll RA (1990b) Physiological properties of excitatory synaptic transmission in the central nervous system. Cold Spring Harbor Symp Quant Biol 55:87-93.

Hirsch YC, Crépel F (1990) Use-dependent changes in synaptic efficacy in rat prefrontal neurons in vitro. J Physiol (Lond) 427:31-49.

Holt GR, Koch C (1997) Shunting inhibition does not have a divisive effect on firing rates. Neural Comput 9:1001-1013.

Jahr CE, Stevens CF (1990) Voltage dependence of NMDA-activated macroscopic conductances predicted by single-channel kinetics. J Neurosci 10:3178-3182.

Javitt DC, Zukin SR (1991) Recent advances in the phencyclidine model of schizophrenia. Am J Psychiatry 148:1301-1308.

Kang Y (1995) Differential paired pulse depression of non-NMDA and NMDA currents in pyramidal cells of the rat frontal cortex. J Neurosci 15:8268-8280.

Kritzer MF, Goldman-Rakic PS (1995) Intrinsic circuit organization of the major layers and sublayers of the dorsolateral prefrontal cortex in the rhesus monkey. J Comp Neurol 359:131-143.

Krystal JH, Karper LP, Seibyl JP, Freeman GK, Delaney R, Bremmer JD, Heninger GR, Bowers MB, Charney DS (1994) Subanesthetic effects of the noncompetitive NMDA antagonist, ketamine, in humans. Arch Gen Psychiatry 51:199-214.

Kubota K, Niki H (1971) Prefrontal cortical unit activity and delayed alternation performance in monkeys. J Neurophysiol 34:337-347.

Larson-Prior LJ, Ulinski PS, Slater NT (1991) Excitatory amino acid receptor-mediated transmission in geniculocortical and intracortical pathways within visual cortex. J Neurophysiol 66:293-306.

Lester RA, Clements JD, Westbrook GL, Jahr CE (1990) Channel kinetics determine the time course of NMDA receptor-mediated synaptic currents. Nature 346:565-567.

Levitt B, Lewis DA, Yoshioka T, Lund J (1993) Topography of pyramidal neuron intrinsic connections in macaque monkey prefrontal cortex (areas 9 and 46). J Comp Neurol 338:360-376.

Lisman JE, Fellous J-M, Wang X-J (1998) A role for NMDA-receptor channels in working memory. Nat Neurosci 1:273-275.

Liu YH, Wang X-J (1999) Adaptation of cortical pyramidal neurons to stochastic inputs. submitted.

Macdonald RL, Olsen RW (1994) GABA receptor channels. Annu Rev Neurosci 17:569-602.

Mainen ZF, Malinow R, Svoboda K (1999) Synaptic calcium transients in single spines indicate that NMDA receptors are not saturated. Nature 399:151-155.

Markram H, Tsodyks M (1996) Redistribution of synaptic efficacy between neocortical pyramidal neurons. Nature 382:807-810.

Markram H, Lübke J, Frotscher M, Roth A, Sakmann B (1997) Physiology and anatomy of synaptic connections between thick tufted pyramidal neurones in the developing rat neocortex. J Physiol (Lond) 500:409-440.

Mason A, Larkman A (1990) Correlations between morphology and electrophysiology of pyramidal neurons in slices of rat visual cortex. II. electrophysiology. J Neurosci 10:1415-1428.

McCormick DA, Connors BW, Lighthall JW, Prince DA (1985) Comparative electrophysiology of pyramidal and sparsely spiny stellate neurons of the neocortex. J Neurophysiol 54:782-806.

Miller EK, Erickson CA, Desimone R (1996) Neural mechanisms of visual working memory in prefrontal cortex of the macaque. J Neurosci 16:5154-5167.

Miyashita Y, Chang HS (1988) Neuronal correlate of pictorial shortterm memory in the primate temporal cortex. Nature 331:68-70.

Moody SL, Wise SP, di Pellegrino G, Zipser D (1998) A model that accounts for activity in primate frontal cortex during a delayed matching-to-sample task. J Neurosci 18:399-410.

Rainer G, Assad WF, Miller EK (1998) Memory fields of neurons in the primate prefrontal cortex. Proc Natl Acad Sci USA 95:15008-15013.

Rao SG, Williams GV, Goldman-Rakic PS (1999) Isodirectional tuning of adjacent interneurons and pyramidal cells during working memory: evidence for microcolumnar organization in PFC. J Neurophysiol 81:1903-1916.

Ricciardi LM (1977) Diffusion processes and related topics in biology. Berlin: Springer.

Rinzel J, Ermentrout GB (1998) In: Methods in neuronal modeling Ed 2 (Koch C, Segev I, eds), pp 251-291. Cambridge, MA: MIT.

Romanides AJ, Duffy P, Kalvas PW (1999) Glutamatergic and dopaminergic afferents to the prefrontal cortex regulate spatial working memory in rats. Neuroscience 92:97-106.

Romo R, Brody CD, Hernández A, Lemus L (1999) Neuronal correlates of parametric working memory in the prefrontal cortex. Nature 399:470-474.

Scherzer CR, Landwehrmeyer GB, Kerner JA, Counihan TJ, Kosinski CM, Standaert DG, Daggett LP, Velicelebi G, Penney Jr JB, Young AB (1998) Expression of $N$-Methyl-D-Aspartate receptor subunit mRNAs in the human brain: hippocampus and cortex. J Comp Neurol 390:75-90.

Seung S (1996) How the brain keeps the eyes still. Proc Natl Acad Sci USA 93:13339-13344.

Shadlen MN, Newsome WT (1994) Noise, neural codes and cortical organization. Curr Opin Neurobiol 4:569-579.

Stevens CF, Wang Y (1995) Facilitation and depression at single central synapses. Neuron 14:795-802.

Strogatz SH (1994) Nonlinear dynamics and chaos. Reading, MA: Addison-Wesley.

Sutor B, Hablitz JJ (1989) EPSPs in rat neocortical neurons in vitro II. involvement of $N$-methyl-D-aspartate receptors in the generation of EPSPs. J Neurophysiol 61:621-634.

Thomson AM, Deuchars J (1995) Diverse pre- and post-synaptic properties of fast excitatory synapses. In: Excitatory amino acids and synaptic transmission, Ed 2 (Wheal HV, Thomson AV, eds) pp 145-172. London: Academic. 
Thomson AM, West DC, Lodge D (1985) An N-methyl-D-aspartate receptor mediated synapse in rat cerebral cortex: a site of action of ketamine? Nature 313:479-481.

Treves A (1993) Mean-field analysis of neuronal spike dynamics. Network 4:259-284.

Troyer TW, Miller KD (1997) Physiological gain leads to high ISI variability in a simple model of a cortical regular spiking cell. Neural Comput 9:971-983.

Tuckwell H (1988) Introduction to theoretical neurobiology, Vol 2. Cambridge, England: Cambridge UP.

van Vreeswijk C, Hansel D (1997) Rhythmic bursting in networks of adaptive spiking neurons. Abstract at the Sixth Annual Computational Neuroscience Meeting, CNS*97, Big Sky, MT.

van Vreeswijk C, Sompolinski H (1996) Chaos in neuronal networks with balanced excitatory and inhibitory activity. Science 274:1724-1726.

Verma A, Moghaddam B (1996) NMDA receptor antagonists impair prefrontal cortex function as assessed via spatial delayed alternation performance in rats: modulation by dopamine. J Neurosci 16: 373-379.

Wang X-J (1998) Calcium coding and adaptive temporal computation in cortical pyramidal neurons. J Neurophysiol 79:1549-1566.

Wang X-J (1999) Fast burst firing and short-term synaptic plasticity: a model of neocortical chattering neurons. Neuroscience 89:347-362.

Wang X-J, Rinzel J (1992) Alternating and synchronous rhythms in reciprocally inhibitory model neurons. Neural Comput 4:84-97.

Weinberger DR, Berman KF (1996) Prefrontal function in schizophrenia: confounds and controversies. Philos Trans R Soc Lond B Biol Sci 351:1495-1503.

Wilson H, Cowan J (1973) A mathematical theory of the functional dynamics of cortical and thalamic nervous tissue. Kybernetik 13:55-80. Zipser D, Kehoe B, Littlewort G, Fuster J (1993) A spiking network of short-term active memory. J Neurosci 13:3406-3420. 